\title{
A Mayer-Vietoris formula for persistent homology with an application to shape recognition in the presence of occlusions
}

\author{
Barbara Di Fabio ${ }^{1,3}$ Claudia Landi ${ }^{2,3, *}$ \\ ${ }^{1}$ Dipartimento di Matematica, Università di Bologna, Italia \\ ${ }^{2}$ Dipartimento di Scienze e Metodi dell’Ingegneria, Università di Modena e Reggio Emilia, Italia \\ ${ }^{3}$ ARCES, Università di Bologna, Italia
}

July 14, 2010

\begin{abstract}
In algebraic topology it is well-known that, using the Mayer-Vietoris sequence, the homology of a space $X$ can be studied splitting $X$ into subspaces $A$ and $B$ and computing the homology of $A, B, A \cap B$. A natural question is to which an extent persistent homology benefits of a similar property. In this paper we show that persistent homology has a Mayer-Vietoris sequence that in general is not exact but only of order two. However, we obtain a Mayer-Vietoris formula involving the ranks of the persistent homology groups of $X, A, B$ and $A \cap B$ plus three extra terms. This implies that topological features of $A$ and $B$ either survive as topological features of $X$ or are hidden in $A \cap B$. As an application of this result, we show that persistence diagrams are able to recognize an occluded shape by showing a common subset of points.
\end{abstract}

Keywords: Čech homology, Mayer-Vietoris sequence, persistence diagram, partial matching, shape occlusion

MSC (2010): 55N05, 68U05

\section{Introduction}

Shape matching and retrieval are key aspects in the design of search engines based on visual, rather than keyword, information. Generally speaking, shape matching methods rely on the computation of a shape description, also called a signature, that effectively captures some essential features of the object.

Persistent homology is a method for shape description and matching that is suitable for any multidimensional data set that can be modeled as a topological space $X$, and whose shape properties can be described by a continuous function $\varphi$ defined on it (e.g., a domain of $\mathbb{R}^{2}$ and the height function may model terrain elevations). Persistent homology groups are defined in terms of lifetime of homology classes along the filtration of $X$ given by the lower level sets of $\varphi$. Surveys on persistent homology include those by Edelsbrunner and Harer [9] and Carlsson [2]. In Biasotti et al. [1] persistent homology is described in relation to other methods that are grounded in Morse theory.

A natural question concerning persistent homology groups is whether there is some sort of Mayer-Vietoris formula applicable to their ranks. In algebraic topology, the Mayer-Vietoris formula is a powerful tool for studying the homology of a space $X$ by splitting it into subspaces whose homology is simpler to compute. So, on the one hand, the interest in an analog of the Mayer-Vietoris formula also in persistent homology stems from the

${ }^{*}$ Corresponding author. E-mail Address: clandi@unimore.it 
wish of developing a similar procedure to compute persistent homology groups. On the other hand, it also seems the right tool to deal with partial matching problems.

The ability to perform not only global matching, but also partial matching, is regarded as one of the most meaningful properties in order to evaluate the performance of a shape matching method (cf., e.g., [23]). Persistent homology groups, like most methods of their class, work on a shape as a whole. In general, it is argued that global object methods are not well suited for partial matching, whereas methods based on computing local features may be more suited to this task.

In this paper we tackle the problem of finding a Mayer-Vietoris formula for persistent homology groups. We show that persistent homology has a Mayer-Vietoris sequence that in general is not exact but only of order two. Nevertheless, we present a Mayer-Vietoris formula for persistent homology groups of a space $X$ that is the union of two subspaces $A$ and $B$, which involves the ranks of the persistent homology groups of $X, A, B$ and $A \cap B$ plus three extra terms. Furthermore, we prove that, when persistent homology groups are represented through the so-called persistence diagrams as collections of points in the plane, the persistence diagrams of $A$ and $B$ leave a fingerprint in the persistence diagram of $X$. Indeed, persistence diagrams are able to detect a partial matching between two shapes by showing a common subset of points.

As an application of partial matching detection, we consider the problem of shape recognition in the presence of occlusions. Basically, the interest in robustness against partial occlusions is motivated by the problem of recognizing an object partially hidden by some other foreground object in the same image. The ability to recognize shapes, even when they are partially occluded by another pattern, has been investigated in the Computer Vision literature by various authors, with reference to a variety of shape recognition methods (see, e.g., $[4,13,15,19,21,22])$. We experimentally show that the ranks of the 0th degree persistent homology groups, also known as size function [11], are able to preserve local information, so that they can manage uncertainty due to the presence of occluded shapes.

The paper is organized as follows. In Section 2 we recall background notions about persistent homology in the Čech homology setting. In Section 3 we introduce the Mayer-Vietoris sequence of persistent Čech homology groups and prove our main result concerning the Mayer-Vietoris relationship among the persistent homology groups of $X, A, B$, and $A \cap B$ in terms of their ranks. Section 4 is devoted to the consequent relationship among points of persistence diagrams in terms of their coordinates and multiplicities. Before concluding the paper with a brief discussion of our results, we show some experiments in Section 5 related to the shape recognition problem in the presence of occlusions, thus demonstrating the usefulness of our Mayer-Vietoris formula in partial matching problems. Any reader not familiar with Čech homology and the Mayer-Vietoris sequence can find a brief survey of the subject in Appendix A. The proofs of some non-central results are deferred to Appendix B.

\section{Background on persistent homology}

In this section we give a brief review of concepts related to persistent homology that will be used in the sequel. Following [3], each topological space considered here will be assumed to be triangulable, i.e., homeomorphic to the body of a finite simplicial complex, and endowed with a continuous real-valued function. This function is used to construct a filtration of the space aimed at capturing its topological features.

Given a space $X$ and a continuous function $\varphi: X \rightarrow \mathbb{R}$, for every $u \in \mathbb{R}$, we denote by $X_{u}$ the lower level set $\{P \in X: \varphi(P) \leq u\}$. The sets $X_{u}, u \in \mathbb{R}$, give a filtration of $X$. Since $\varphi$ is just continuous, $X_{u}$ is not necessarily triangulable.

We use the following notations: $\Delta^{*}=\left\{(u, v) \in \mathbb{R}^{2}: u<v\right\} \cup\{(u, \infty): u \in \mathbb{R}\}, \Delta$ is the diagonal of $\mathbb{R}^{2}$, and $\overline{\Delta^{*}}=\Delta^{*} \cup \Delta$. In the sequel, points $(u, v) \in \Delta^{*}$ such that $v<\infty$ are called proper points; points $(u, v) \in \Delta^{*}$ such that $v=\infty$ are said to be points at infinity.

Given a proper point $(u, v) \in \Delta^{*}$, let $\imath^{u, v}$ be the inclusion of $X_{u}$ into $X_{v}$. This mapping induces a homomorphism of Čech homology groups $\imath_{k}^{u, v}: \check{H}_{k}\left(X_{u}\right) \rightarrow \breve{H}_{k}\left(X_{v}\right)$ for each integer $k$, leading to the definition of persistent $(\check{C}$ ech ) homology groups. 
Definition 2.1. Let $X$ be a triangulable space, and $\varphi: X \rightarrow \mathbb{R}$ a continuous function. Given a proper point $(u, v) \in \Delta^{*}$, the $k$ th persistent (Čech ) homology group $\check{H}_{k}^{u, v}(X, \varphi)$ is the image of the homomorphism $\imath_{k}^{u, v}$ between $k$ th $\breve{C}$ ech homology groups induced by the inclusion mapping of $X_{u}$ into $X_{v}: \check{H}_{k}^{u, v}(X, \varphi)=\operatorname{im} \imath_{k}^{u, v}$.

Throughout the paper the homology coefficients will be taken in a vector space over a field. In this way, from [10], we know that $\breve{H}_{k}^{u, v}(X, \varphi)$ is a vector space over the same field. Hence, persistent homology groups are torsion free and thus completely determined by their rank. In plain words, the rank of the $k$-persistent homology group at $(u, v)$, denoted by $\operatorname{rk} \breve{H}_{k}^{u, v}(X, \varphi)$, counts the maximum number of independent non-bounding $k$-cycles born before $u$ and still alive at $v$, according to the filtration of $X$ constituted by the lower level sets of $\varphi$.

Given a space $X$ filtered by $\varphi$, a global description of all the groups $\breve{H}_{k}^{u, v}(X, \varphi)$, with $(u, v)$ a proper point varying in $\Delta^{*}$, is provided by the so-called persistence diagrams (see Definition 2.4). These are multisets of points (i.e., sets of points equipped with a multiplicity) of $\overline{\Delta^{*}}$. Before giving the definition of a persistence diagram, we recall the definitions of multiplicity of a proper point and multiplicity of a point at infinity.

Definition 2.2. Let $k \in \mathbb{Z}$ and let $p=(\bar{u}, \bar{v}) \in \Delta^{*}$ be a proper point. The multiplicity $\mu_{(X, \varphi), k}(p)$ of $p$ is the finite non-negative number defined by

$$
\lim _{\varepsilon \rightarrow 0^{+}}\left(\operatorname{rk} \breve{H}_{k}^{\bar{u}+\varepsilon, \bar{v}-\varepsilon}(X, \varphi)-\operatorname{rk} \check{H}_{k}^{\bar{u}-\varepsilon, \bar{v}-\varepsilon}(X, \varphi)-\operatorname{rk} \breve{H}_{k}^{\bar{u}+\varepsilon, \bar{v}+\varepsilon}(X, \varphi)+\operatorname{rk} \breve{H}_{k}^{\bar{u}-\varepsilon, \bar{v}+\varepsilon}(X, \varphi)\right) .
$$

Definition 2.3. Let $k \in \mathbb{Z}$ and let $r$ be the vertical line with equation $u=\bar{u}$ in the plane $u, v$, identified with the point at infinity $(\bar{u}, \infty) \in \Delta^{*}$. The multiplicity $\mu_{(X, \varphi), k}(r)$ of $r$ is the finite non-negative number defined by

$$
\lim _{\varepsilon \rightarrow 0^{+}}\left(\operatorname{rk} \check{H}_{k}^{\bar{u}+\varepsilon, 1 / \varepsilon}(X, \varphi)-\operatorname{rk} \check{H}_{k}^{\bar{u}-\varepsilon, 1 / \varepsilon}(X, \varphi)\right) .
$$

Points with a positive multiplicity are particularly interesting. Indeed, the abscissa and the ordinate of a proper point $p$ with $\mu_{(X, \varphi), k}(p)>0$ identify, respectively, the level at which a new $k$-homology class is created and the level at which it is annihilated through the filtration. The abscissa of a point at infinity $r$ with $\mu_{(X, \varphi), k}(r)>0$ encodes the level at which a new $k$-homology class of $X$ is born. The real values at which the lower level sets undergo a topological change are called homological critical values (see Definition B.1). This intuition about the link between points with a positive multiplicity and homological critical values needs some attention, and is treated in detail in Appendix B.

Let us observe that under our mild assumption that $\varphi$ is just continuous, infinitely many proper points with a positive multiplicity can occur, accumulating onto the diagonal of $\mathbb{R}^{2}$. An example of this phenomenon is illustrated in Figure 5. Instead, when stronger assumptions on $\varphi$ are made, only finitely many of these points exist. For this reason, our definitions of multiplicity slightly differ from the one given in [5].

Definition 2.4. The persistence diagram $D_{k}(X, \varphi)$ is the multiset of all points $(u, v) \in \Delta^{*}$ such that $\mu_{(X, \varphi), k}(u, v)>$ 0 , counted with their multiplicity, union the points of $\Delta$, counted with infinite multiplicity.

Obviously, the knowledge of the persistent homology groups allows one to compute the associated persistence diagrams. It is worth emphasizing that the converse is also true, thus clarifying the importance of persistence diagrams in persistent homology. This is precisely stated by the following theorem.

Theorem 2.5. For every proper point $(\bar{u}, \bar{v}) \in \Delta^{*}$, and $k \in \mathbb{Z}$, we have

$$
\operatorname{rk} \breve{H}_{k}^{\bar{u}, \bar{v}}(X, \varphi)=\sum_{\substack{(u, v) \in \Delta * \\ u \leq \bar{u}, v>\bar{v}}} \mu_{(X, \varphi), k}(u, v) .
$$

An example of persistence diagram in 0th homology degree is displayed in Figure 1. We recall that, in the case $k=0, \operatorname{rk} \breve{H}_{k}^{u, v}(X, \varphi)$ counts the number of connected components born before the level $u$ and still alive at level $v$. In this example we consider a curve $X$ of $\mathbb{R}^{2}$ represented by the solid line in Figure 1(A), and the function 


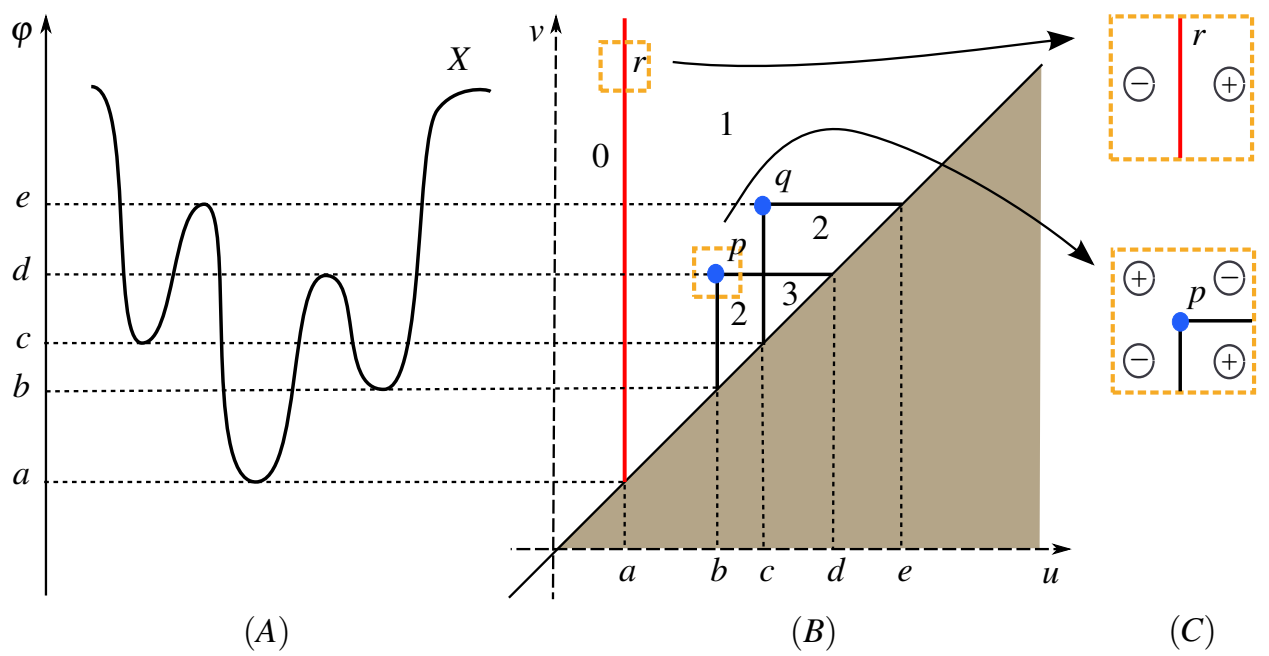

Figure 1: (A) A curve $X \subseteq \mathbb{R}^{2}$ represented by a continuous line, and the function $\varphi: X \rightarrow \mathbb{R}$ such that $\varphi(P)=y$ for every $P=(x, y) \in X$. $(B)$ The points (proper and at infinity) of the persistence diagram $D_{0}(X, \varphi) .(C)$ Computation of multiplicities seen through lens.

$\varphi: X \rightarrow \mathbb{R}$ that associates with each point $P \in X$ its ordinate in the plane. The sole points (both proper and at infinity) of the associated persistence diagram $D_{0}(X, \varphi)$ are $p, q$, and $r$, and are shown in Figure $1(B)$. Here, solid lines divide $\Delta^{*}$ into regions where the rank of the 0th persistence homology group is constant. The value displayed in each region is the value taken by the rank of 0th persistence homology groups for $(u, v)$ varying in that region. For instance, when $c \leq v<d, X_{v}$ has three connected components. Only one of them contains at least one point of $X_{u}$, when $a \leq u<b$; two of them contain at least one point of $X_{u}$, when $b \leq u<c$; all of them contain at least one point of $X_{u}$, when $c \leq u<v<d$. Therefore, when $c \leq v<d$, $\operatorname{rk} \breve{H}_{0}^{u, v}(X, \varphi)=1$ for $a \leq u<b$; $\operatorname{rk} \breve{H}_{0}^{u, v}(X, \varphi)=2$ for $b \leq u<c ; \operatorname{rk} \breve{H}_{0}^{u, v}(X, \varphi)=3$ for $c \leq u<v$.

Figure $1(C)$ zooms in on two points of the persistence diagram (one proper $p$, and one at infinity $r$ ) to explain how their multiplicity is computed. The alternating sum of the rank of the 0th persistent homology group at four points around $p$ is $2-1-1+1$, giving $\mu_{(X, \varphi), 0}(p)=1$. The alternating sum of the rank of the 0th persistent homology group at two points next to $r$ is $1-0$, giving $\mu_{(X, \varphi), 0}(r)=1$.

Let us conclude this section with some observations.

The fact that persistence diagrams uniquely determine persistent homology groups allows one to translate the comparison between persistent homology groups into distances between multisets of points, in a way that is robust against deformations $[3,5,18]$.

The motivation for using Čech homology rather than a more ordinary homology theory is given by the following fact, that is a consequence of the continuity axiom (see Appendix A).

Remark 2.6. Using singular rather than Čech homology causes Theorem 2.5 to be valid only outside a set of zero measure.

We emphasize that, in our setting, Čech homology satisfies all the ordinary homological axioms (see Appendix A), so that it can be used in the same way as the singular and simplicial theories.

\section{The Mayer-Vietoris sequence of persistent homology groups}

In this section, we look for a relation expressing the rank of the persistent homology groups of $X$ filtered by $\varphi$ in terms of the ranks of the persistent homology groups of two subspaces $A$ and $B$ filtered by $\varphi_{\mid A}$ and $\varphi_{\mid B}$, respectively. We require $A$ and $B$ to be triangulable subsets of $X$ such that $X=A \cup B$ and $A \cap B$ is triangulable. 
Let us observe that the functions $\varphi_{\mid A \cap B}, \varphi_{\mid A}$, and $\varphi_{\mid B}$ are continuous, as restrictions of the continuous function $\varphi: X \rightarrow \mathbb{R}$ to spaces endowed with the relative topology induced by the topology of $X$. This justifies the choice of taking $\varphi$ just continuous. These hypotheses on $X, A, B$ and $A \cap B$ will be maintained throughout the paper. Moreover, since from now on $\varphi: X \rightarrow \mathbb{R}$ is fixed, we will simply write $\operatorname{rk} \breve{H}_{k}^{u, v}(X), D_{k}(X)$, and $\mu_{X, k}$ instead of $\operatorname{rk} \check{H}_{k}^{u, v}(X, \varphi), D_{k}(X, \varphi)$, and $\mu_{(X, \varphi), k}$, respectively. Analogously for $A, B$, and $A \cap B$, since also $\varphi_{\mid A}, \varphi_{\mid B}, \varphi_{\mid A \cap B}$ are fixed.

Our main tool for finding such a relation is the Mayer-Vietoris sequence of the triad $(X, A, B)$ :

$$
\cdots \longrightarrow \check{H}_{k+1}(X) \stackrel{\delta_{k}}{\longrightarrow} \check{H}_{k}(A \cap B) \stackrel{\alpha_{k}}{\longrightarrow} \check{H}_{k}(A) \oplus \check{H}_{k}(B) \stackrel{\beta_{k}}{\longrightarrow} \check{H}_{k}(X) \stackrel{\delta_{k-1}}{\longrightarrow} \cdots \longrightarrow \check{H}_{0}(X) \longrightarrow 0,
$$

where $\delta_{k}$ is the homomorphism $\check{H}_{k+1}(X) \ni[z] \mapsto\left[\partial\left(z_{\left.\right|_{A}}\right)\right] \in \breve{H}_{k}(A \cap B), \alpha_{k}$ is the homomorphism $\breve{H}_{k}(A \cap B) \ni$ $[z] \mapsto([z],[-z]) \in \check{H}_{k}(A) \oplus \check{H}_{k}(B)$, and $\beta_{k}$ is the homomorphism $\check{H}_{k}(A) \oplus \check{H}_{k}(B) \ni\left([z],\left[z^{\prime}\right]\right) \mapsto\left[z+z^{\prime}\right] \in \check{H}_{k}(X)$. Intuitively, the homomorphisms $\delta_{k}, \alpha_{k}$, and $\beta_{k}$ are described in Figure 2 for the case $k=0$.
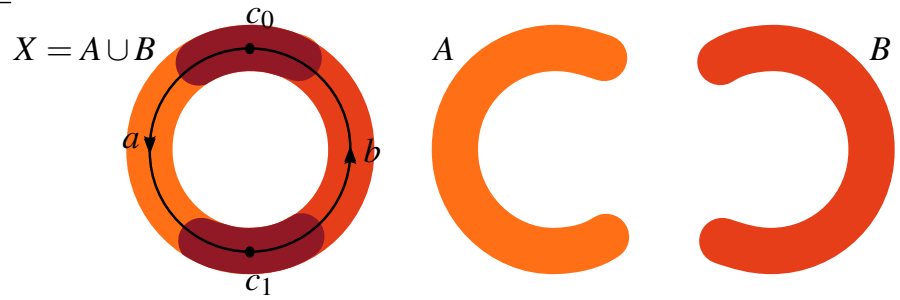

Figure 2: For this triad $(X, A, B), a+b$ is a 1-cycle in $X, c_{0}$ and $c_{1}$ are 0 -cycles in $A \cap B$ (and hence also in $A$ and $B$ ). Moreover, $c_{0}$ and $c_{1}$ are cobordant in $A$ and $B$ but not in $A \cap B$. We have that $\delta_{0}([a+b])=\left[c_{1}-c_{0}\right], \alpha_{0}\left(\left[c_{i}\right]\right)=\left(\left[c_{i}\right],-\left[c_{i}\right]\right), \beta_{0}\left(\left[c_{i}\right],\left[c_{j}\right]\right)=\left[c_{i}\right]+\left[c_{j}\right]$.

Under our assumptions, the Mayer-Vietoris sequence above is exact (see Appendix A). It is well known that the exactness of such a sequence provides a relation among the ranks of $\breve{H}_{k}(X), \breve{H}_{k}(A), \breve{H}_{k}(B), \breve{H}_{k}(A \cap B)$, and of the kernels of homomorphisms $\alpha_{k}$ (see, e.g., $[8,10]$ ):

$$
\operatorname{rk} \check{H}_{k}(X)=\operatorname{rk} \check{H}_{k}(A)+\operatorname{rk} \check{H}_{k}(B)-\operatorname{rk} \check{H}_{k}(A \cap B)+\operatorname{rkker} \alpha_{k}+\operatorname{rkker} \alpha_{k-1} .
$$

This relation can be easily checked in the example of Figure 2.

The novelty of our approach is the study of Mayer-Vietoris sequences for different triads of lower level sets $\left(X_{u}, A_{u}, B_{u}\right)$, interlacing them with long exact sequences of the pair (diagram (2)). This involves considering also a relative Mayer-Vietoris sequence (see Appendix A). In this way, we are able to generalize formula (1) to persistent homology (Theorem 3.7).

We shall apply this Mayer-Vietoris formula for persistent homology in the next section in order to show that it is possible to match a subset of the points of $D_{k}(X)$ to points of either $D_{k}(A)$ or $D_{k}(B)$, under suitable conditions.

We begin by underlining some simple properties of the lower level sets of $X, A, B$, and $A \cap B$. Then we show that there exists a Mayer-Vietoris sequence for persistent homology groups that is of order 2 (Proposition 3.4), and that, under proper assumptions, it induces a short exact sequence involving the $k$ th persistent homology groups of $X, A, B$, and $A \cap B$ (Proposition 3.9).

Lemma 3.1. Let $u \in \mathbb{R}$. Let us endow $X_{u}$ with the relative topology induced by the topology of $X$. Then $A_{u}$ and $B_{u}$ are closed sets in $X_{u}$. Moreover, $X_{u}=A_{u} \cup B_{u}$ and $A_{u} \cap B_{u}=(A \cap B)_{u}$.

Proof. Let us recall that the relative topology induced on $X_{u}$ by the topology of $X$ is that in which the open (closed, respectively) sets are the intersections with $X_{u}$ of open (closed, respectively) sets of $X$ [10]. Therefore, $A_{u}$ is closed in $X_{u}$ since $A_{u}=A \cap X_{u}$, with $A$ triangulable, and hence closed in $X$. Analogously for $B_{u}$. Showing that $X_{u}=A_{u} \cup B_{u}$ and $A_{u} \cap B_{u}=(A \cap B)_{u}$ is trivial. 
Lemma 3.1 ensures that, for every proper point $(u, v) \in \Delta^{*}$, we can consider the following diagram:

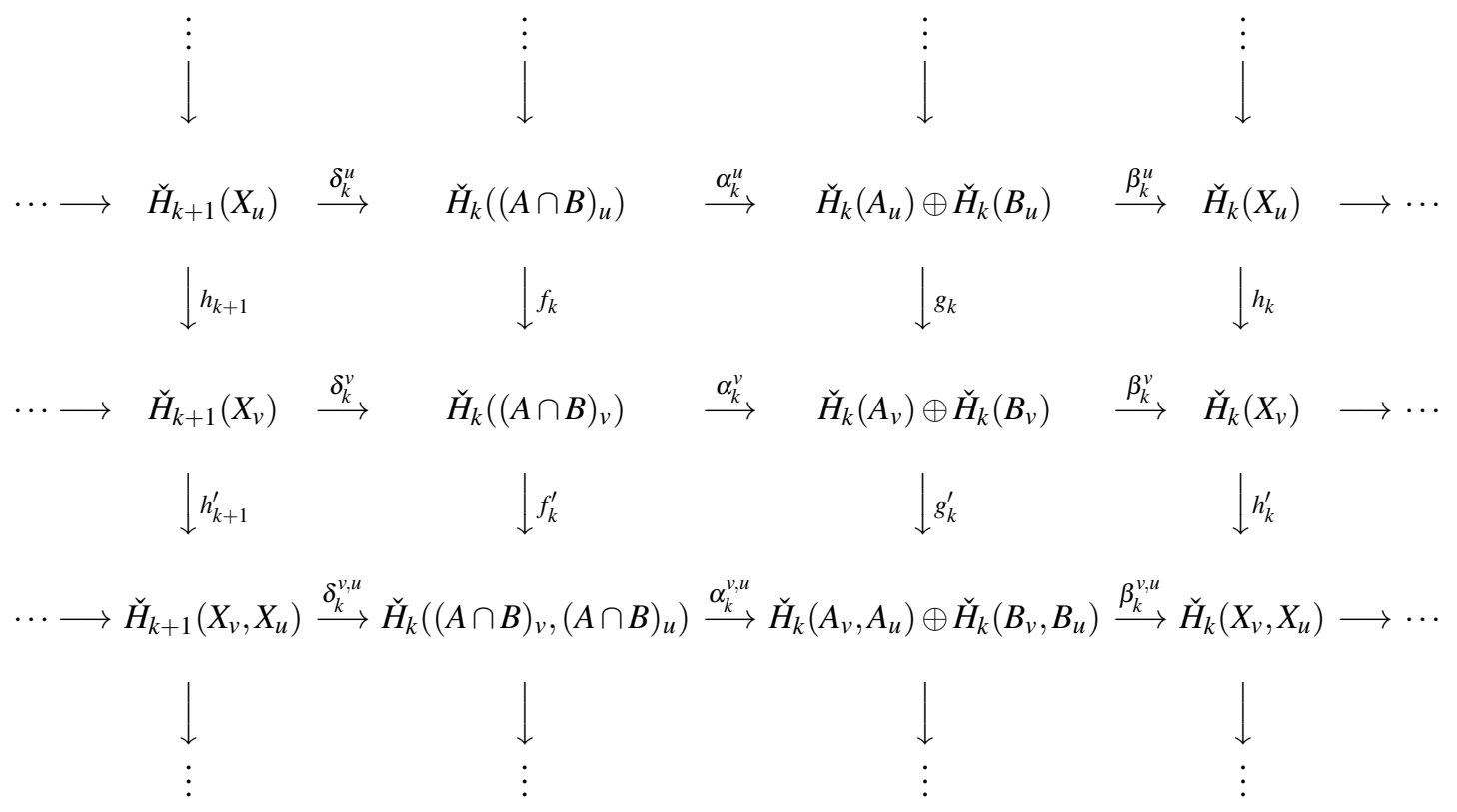

where the top horizontal line belongs to the Mayer-Vietoris sequence of the triad $\left(X_{u}, A_{u}, B_{u}\right)$, the second horizontal line belongs to the Mayer-Vietoris sequence of the triad $\left(X_{v}, A_{v}, B_{v}\right)$, and the third one belongs to the relative Mayer-Vietoris sequence of the triad $\left(\left(X_{v}, X_{u}\right),\left(A_{v}, A_{u}\right),\left(B_{v}, B_{u}\right)\right)$. The leftmost and rightmost vertical lines belong to the long sequence of the pair $\left(X_{v}, X_{u}\right)$, the second vertical line belongs to the long sequence of the pair $\left(A \cap B_{v}, A \cap B_{u}\right)$, and the third one belongs to the direct sum of the long sequences of the pairs $\left(A_{v}, A_{u}\right)$ and $\left(B_{v}, B_{u}\right)$.

Lemma 3.2. Each vertical and horizontal line in diagram (2) is exact. Moreover, each square in the same diagram is commutative.

Proof. We recall that we are assuming that $X$ is compact and $\varphi$ continuous, therefore $X_{u}$ and $X_{v}$ are compact, as well as $A_{u}, A_{v}, B_{u}$ and $B_{v}$ by Lemma 3.1. Moreover, since we are also assuming that the coefficient group is a vector space over a field, it holds that the homology sequences of the pairs $\left(X_{v}, X_{u}\right),\left((A \cap B)_{v},(A \cap B)_{u}\right),\left(A_{v}, A_{u}\right)$, $\left(B_{v}, B_{u}\right)$ (vertical lines) are exact (cf. Theorem A.1 in Appendix A).

Analogously, the Mayer-Vietoris sequences of $\left(X_{u}, A_{u}, B_{u}\right)$ and $\left(X_{v}, A_{v}, B_{v}\right)$, and the relative Mayer-Vietoris sequence of $\left(\left(X_{v}, X_{u}\right),\left(A_{v}, A_{u}\right),\left(B_{v}, B_{u}\right)\right)$ (horizontal lines) are exact (cf. Theorems A.2 and A.4 in Appendix A).

About the commutativity of the top squares, it is sufficient to apply Theorem A.3 in Appendix A. The same conclusion can be drawn for the commutativity of the bottom squares, with $X_{v}$ replaced by $\left(X_{v}, \emptyset\right), A_{v}$ by $\left(A_{v}, \emptyset\right)$ and $B_{v}$ by $\left(B_{v}, \emptyset\right)$, respectively, applying Theorem A.5.

Remark 3.3. For every $k \in \mathbb{Z}$, and every proper point $(u, v) \in \Delta^{*}, \operatorname{im} f_{k}=\check{H}_{k}^{u, v}(A \cap B), \operatorname{im} g_{k}=\check{H}_{k}^{u, v}(A) \oplus \check{H}_{k}^{u, v}(B)$, and $\operatorname{im} h_{k}=\check{H}_{k}^{u, v}(X)$.

The following proposition proves that the commutativity of squares in diagram (2) induces a Mayer-Vietoris sequence of order 2 involving the $k$ th persistent homology groups of $X, A, B$, and $A \cap B$, for every integer $k$.

Proposition 3.4. Let us consider the sequence of homomorphisms of persistent homology groups

$$
\cdots \longrightarrow \check{H}_{k+1}^{u, v}(X) \stackrel{\delta}{\longrightarrow} \check{H}_{k}^{u, v}(A \cap B) \stackrel{\alpha}{\longrightarrow} \check{H}_{k}^{u, v}(A) \oplus \check{H}_{k}^{u, v}(B) \stackrel{\beta}{\longrightarrow} \check{H}_{k}^{u, v}(X) \longrightarrow \cdots
$$


where $\delta=\delta_{k \mid \operatorname{im} h_{k+1}}, \alpha=\alpha_{k \mid \operatorname{im}_{f_{k}}^{v}}$, and $\beta=\beta_{k \mid \operatorname{im} g_{k}}$. The following statements hold:

(i) $\operatorname{im} \delta \subseteq \operatorname{ker} \alpha$;

(ii) $\operatorname{im} \alpha \subseteq \operatorname{ker} \beta$;

(iii) $\operatorname{im} \beta \subseteq \operatorname{ker} \delta$,

that is, the sequence is of order 2.

Proof. First of all, we observe that, by Lemma 3.2, $\operatorname{im} \delta \subseteq \operatorname{im} f_{k}, \operatorname{im} \alpha \subseteq \operatorname{im} g_{k}$ and $\operatorname{im} \beta \subseteq \operatorname{im} h_{k}$. Now we prove only claim (i), considering that (ii) and (iii) can be deduced analogously. Let $c \in \operatorname{im} \delta$. Then $c \in \operatorname{im} f_{k}$ and $c \in \operatorname{im} \delta_{k}^{v}=\operatorname{ker} \alpha_{k}^{v}$. Therefore $c \in \operatorname{ker} \alpha$.

\subsection{The persistent homology group of the union of two spaces}

This subsection is devoted to provide a Mayer-Vietoris formula for persistent homology (Theorem 3.7). It is based on the lemma below, that holds for any exact sequence of homomorphisms of finitely generated groups (Lemma 3.5). For this reason, from now on, all the considered lower level sets will be assumed to have finitely generated homology groups.

Lemma 3.5. If $L, M, N, O$ are finitely generated groups, and the sequence of homomorphisms

$$
\cdots \stackrel{\imath}{\longrightarrow} L \stackrel{\lambda}{\longrightarrow} M \stackrel{\mu}{\longrightarrow} N \stackrel{v}{\longrightarrow} O \stackrel{o}{\longrightarrow} \cdots,
$$

is exact, then $\operatorname{rk} N=\operatorname{rk} M-\mathrm{rk} L+\operatorname{rk} \operatorname{ker} \lambda+\operatorname{rk} \operatorname{ker} o$.

Proof. The claim trivially follows from the exactness of the sequence and the dimensional relation linking the domain of each map with its kernel and its image.

Let us observe that the previous relation (1) can be deduced from Lemma 3.5 replacing the long sequence above by the Mayer-Vietoris sequence of the triad $(X, A, B)$.

Applying Lemma 3.5 to the long exact sequence of the pair, we obtain the following result.

Lemma 3.6. For every $k \in \mathbb{Z}$, and every proper point $(u, v) \in \Delta^{*}$, it holds that

$$
\operatorname{rk} \check{H}_{k}^{u, v}(Y)=\operatorname{rk} \check{H}_{k}\left(Y_{v}\right)-\operatorname{rk} \check{H}_{k}\left(Y_{v}, Y_{u}\right)+\operatorname{rk} \check{H}_{k-1}\left(Y_{u}\right)-\operatorname{rk} \check{H}_{k-1}^{u, v}(Y) .
$$

Proof. It is sufficient to consider the long exact sequence of the pair $\left(Y_{v}, Y_{u}\right)$

$$
\cdots \longrightarrow \check{H}_{k}\left(Y_{u}\right) \stackrel{l_{k}}{\longrightarrow} \check{H}_{k}\left(Y_{v}\right) \stackrel{j_{k}}{\longrightarrow} \check{H}_{k}\left(Y_{v}, Y_{u}\right) \stackrel{\partial_{k-1}}{\longrightarrow} \check{H}_{k-1}\left(Y_{u}\right) \stackrel{\imath_{k-1}}{\longrightarrow} \check{H}_{k-1}\left(Y_{v}\right) \stackrel{\partial_{k-2}}{\longrightarrow} \cdots,
$$

and apply Lemma 3.5, with $L$ replaced by $\check{H}_{k}\left(Y_{v}\right), M$ by $\check{H}_{k}\left(Y_{v}, Y_{u}\right), N$ by $\check{H}_{k-1}\left(Y_{u}\right), \lambda$ by $j_{k}$, and $o$ by $\partial_{k-2}$. In this way, we obtain that $\operatorname{rk} \check{H}_{k-1}\left(Y_{u}\right)=\operatorname{rk} \check{H}_{k}\left(Y_{v}, Y_{u}\right)-\operatorname{rk} \check{H}_{k}\left(Y_{v}\right)+\operatorname{rkker} j_{k}+\operatorname{rkker} \partial_{k-2}$. Since, by the exactness, rk ker $j_{k}=\operatorname{rk} \breve{H}_{k}^{u, v}(Y)$, rk ker $\partial_{k-2}=\operatorname{rk} \check{H}_{k-1}^{u, v}(Y)$, reordering the terms, the claim follows.

Theorem 3.7. For every $k \in \mathbb{Z}$, and every proper point $(u, v) \in \Delta^{*}$, it holds that

$$
\operatorname{rk} \check{H}_{k}^{u, v}(X)=\operatorname{rk} \check{H}_{k}^{u, v}(A)+\operatorname{rk} \check{H}_{k}^{u, v}(B)-\operatorname{rk} \check{H}_{k}^{u, v}(A \cap B)+\operatorname{rk} \operatorname{ker} \alpha_{k}^{v}-\operatorname{rk} \operatorname{ker} \alpha_{k}^{v, u}+\operatorname{rk} \operatorname{ker} \alpha_{k-1}^{u} .
$$


Proof. By induction on $k \geq 0$ (for $k<0$ it is trivial).

Case $\boldsymbol{k}=\mathbf{0}$ : Let us consider diagram (2) with $k=0$. Applying Lemma 3.5 to the second horizontal line with $N=\breve{H}_{0}\left(X_{v}\right)$, and to the third one with $N=\breve{H}_{0}\left(X_{v}, X_{u}\right)$, we obtain, respectively, the following equalities:

$$
\begin{gathered}
\operatorname{rk} \check{H}_{0}\left(X_{v}\right)=\operatorname{rk} \check{H}_{0}\left(A_{v}\right) \oplus \check{H}_{0}\left(B_{v}\right)-\operatorname{rk} \check{H}_{0}\left(A_{v} \cap B_{v}\right)+\operatorname{rkker} \alpha_{0}^{v}, \\
\operatorname{rk} \check{H}_{0}\left(X_{v}, X_{u}\right)=\operatorname{rk} \check{H}_{0}\left(A_{v}, A_{u}\right) \oplus \check{H}_{0}\left(B_{v}, B_{u}\right)-\operatorname{rk} \check{H}_{0}\left(A_{v} \cap B_{v}, A_{u} \cap B_{u}\right)+\operatorname{rkker} \alpha_{0}^{v, u} .
\end{gathered}
$$

Now, subtracting equality (4) from equality (3), we have

$$
\begin{aligned}
\operatorname{rk} \check{H}_{0}\left(X_{v}\right)-\operatorname{rk} \check{H}_{0}\left(X_{v}, X_{u}\right)= & \operatorname{rk} \check{H}_{0}\left(A_{v}\right)-\operatorname{rk} \check{H}_{0}\left(A_{v}, A_{u}\right)+\operatorname{rk} \check{H}_{0}\left(B_{v}\right)-\operatorname{rk} \check{H}_{0}\left(B_{v}, B_{u}\right) \\
& -\operatorname{rk} \check{H}_{0}\left((A \cap B)_{v}\right)+\operatorname{rk} \check{H}_{0}\left((A \cap B)_{v},(A \cap B)_{u}\right)+\operatorname{rkker} \alpha_{0}^{v}-\operatorname{rkker} \alpha_{0}^{v, u},
\end{aligned}
$$

which is equivalent to the claimed relation as can be seen by applying Lemma 3.6 with $k=0$ four times with $Y=X, Y=A, Y=B$, and $Y=A \cap B$, respectively.

Inductive step: Let us assume that

$$
\operatorname{rk} \check{H}_{k-1}^{u, v}(X)=\operatorname{rk} \check{H}_{k-1}^{u, v}(A)+\operatorname{rk} \check{H}_{k-1}^{u, v}(B)-\operatorname{rk} \check{H}_{k-1}^{u, v}(A \cap B)+\operatorname{rkker} \alpha_{k-1}^{v}-\operatorname{rkker} \alpha_{k-1}^{v, u}+\operatorname{rkker} \alpha_{k-2}^{u}
$$

Let us apply Lemma 3.5 to the first horizontal line of diagram (2) with $N=\breve{H}_{k-1}\left(X_{u}\right)$, to the second one with $N=\check{H}_{k}\left(X_{v}\right)$, and to the third one with $N=\check{H}_{k}\left(X_{v}, X_{u}\right)$. We respectively obtain

$$
\begin{gathered}
\operatorname{rk} \check{H}_{k-1}\left(X_{u}\right)=\operatorname{rk} \check{H}_{k-1}\left(A_{u}\right) \oplus \check{H}_{k-1}\left(B_{u}\right)-\operatorname{rk} \check{H}_{k-1}\left(A_{u} \cap B_{u}\right)+\operatorname{rkker} \alpha_{k-1}^{u}+\operatorname{rkker} \alpha_{k-2}^{u} . \\
\operatorname{rk} \check{H}_{k}\left(X_{v}\right)=\operatorname{rk} \check{H}_{k}\left(A_{v}\right) \oplus \check{H}_{k}\left(B_{v}\right)-\operatorname{rk} \check{H}_{k}\left(A_{v} \cap B_{v}\right)+\operatorname{rkker} \alpha_{k}^{v}+\operatorname{rkker} \alpha_{k-1}^{v}, \\
\operatorname{rk} \check{H}_{k}\left(X_{v}, X_{u}\right)=\operatorname{rk} \check{H}_{k}\left(A_{v}, A_{u}\right) \oplus \check{H}_{k}\left(B_{v}, B_{u}\right)-\operatorname{rk} \check{H}_{k}\left(A_{v} \cap B_{v}, A_{u} \cap B_{u}\right)+\operatorname{rk} \operatorname{ker} \alpha_{k}^{v, u}+\operatorname{rk} \operatorname{ker} \alpha_{k-1}^{v, u},
\end{gathered}
$$

Now, let us use Lemma 3.6 with $Y=X$ together with the equalities (6), (7), (8), and the inductive assumption (5). We obtain

$$
\begin{aligned}
& \operatorname{rk} \check{H}_{k}^{u, v}(X)=\operatorname{rk} \check{H}_{k}\left(A_{v}\right)+\operatorname{rk} \check{H}_{k}\left(B_{v}\right)-\operatorname{rk} \check{H}_{k}\left(A_{v} \cap B_{v}\right)+\operatorname{rkker} \alpha_{k}^{v}+\operatorname{rkker} \alpha_{k-1}^{v} \\
& -\left(\operatorname{rk} \check{H}_{k}\left(A_{v}, A_{u}\right)+\operatorname{rk} \check{H}_{k}\left(B_{v}, B_{u}\right)-\operatorname{rk} \breve{H}_{k}\left(A_{v} \cap B_{v}, A_{u} \cap B_{u}\right)+\operatorname{rkker} \alpha_{k}^{v, u}+\operatorname{rkker} \alpha_{k-1}^{v, u}\right) \\
& +\operatorname{rk} \check{H}_{k-1}\left(A_{u}\right)+\operatorname{rk} \check{H}_{k-1}\left(B_{u}\right)-\operatorname{rk} \check{H}_{k-1}\left(A_{u} \cap B_{u}\right)+\operatorname{rkker} \alpha_{k-1}^{u}+\operatorname{rkker} \alpha_{k-2}^{u} \\
& -\left(\operatorname{rk} \check{H}_{k-1}^{u, v}(A)+\operatorname{rk} \check{H}_{k-1}^{u, v}(B)-\operatorname{rk} \check{H}_{k-1}^{u, v}(A \cap B)+\operatorname{rkker} \alpha_{k-1}^{v}-\operatorname{rkker} \alpha_{k-1}^{v, u}+\operatorname{rkker} \alpha_{k-2}^{u}\right) \\
& =\operatorname{rk} \breve{H}_{k}\left(A_{v}\right)-\operatorname{rk} \breve{H}_{k}\left(A_{v}, A_{u}\right)+\operatorname{rk} \check{H}_{k-1}\left(A_{u}\right)-\operatorname{rk} \breve{H}_{k-1}^{u, v}(A) \\
& +\operatorname{rk} \check{H}_{k}\left(B_{v}\right)-\operatorname{rk} \check{H}_{k}\left(B_{v}, B_{u}\right)+\operatorname{rk} \check{H}_{k-1}\left(B_{u}\right)-\operatorname{rk} \check{H}_{k-1}^{u, v}(B) \\
& -\left(\operatorname{rk} \check{H}_{k}\left(A_{v} \cap B_{v}\right)-\check{H}_{k}\left(A_{v} \cap B_{v}, A_{u} \cap B_{u}\right)+\operatorname{rk} \check{H}_{k-1}\left(A_{u} \cap B_{u}\right)-\operatorname{rk} \check{H}_{k-1}^{u, v}(A \cap B)\right) \\
& +\operatorname{rkker} \alpha_{k}^{v}-\operatorname{rkker} \alpha_{k}^{v, u}+\operatorname{rkker} \alpha_{k-1}^{u} \text {, }
\end{aligned}
$$

which is equivalent to the claim by Lemma 3.6 applied three times with $Y=A, Y=B, Y=A \cap B$, respectively.

Corollary 3.8. For every $k \in \mathbb{Z}$, and every proper point $(u, v) \in \Delta^{*}$, it holds that

$$
\operatorname{rk} \check{H}_{k}^{u, v}(X)=\operatorname{rk} \check{H}_{k}^{u, v}(A)+\operatorname{rk} \check{H}_{k}^{u, v}(B)-\operatorname{rk} \check{H}_{k}^{u, v}(A \cap B)
$$

if and only if $\mathrm{rk} \operatorname{ker} \alpha_{k}^{v}-\operatorname{rkker} \alpha_{k}^{v, u}+\operatorname{rk} \operatorname{ker} \alpha_{k-1}^{u}=0$.

Proof. Immediate from Theorem 3.7. 
We now show that combining the assumption that all the maps $\alpha_{k}^{v}, \alpha_{k}^{v, u}$ and $\alpha_{k-1}^{u}$ are injective with Proposition 3.4 , there is a short exact sequence involving the $k$ th persistent homology groups of $X, A, B$, and $A \cap B$.

Proposition 3.9. For every $k \in \mathbb{Z}$, and every proper point $(u, v) \in \Delta^{*}$ such that the maps $\alpha_{k}^{v}$, $\alpha_{k}^{v, u}$ and $\alpha_{k-1}^{u}$ are injective, the short sequence

$$
0 \longrightarrow \check{H}_{k}^{u, v}(A \cap B) \stackrel{\alpha}{\longrightarrow} \check{H}_{k}^{u, v}(A) \oplus \check{H}_{k}^{u, v}(B) \stackrel{\beta}{\longrightarrow} \check{H}_{k}^{u, v}(X) \longrightarrow 0
$$

where $\alpha=\alpha_{k \mid \operatorname{im} f_{k}}$ and $\beta=\beta_{k \mid \operatorname{im} g_{k}}^{v}$, is exact.

Proof. By Proposition 3.4, im $\alpha \subseteq \operatorname{ker} \beta$, so we only have to show that $\beta$ is surjective, $\alpha$ is injective, and rk im $\alpha=$ $\operatorname{rkker} \beta$. We recall that, by Remark $3.3, \check{H}_{k}^{u, v}(A \cap B)=\operatorname{im} f_{k}, \check{H}_{k}^{u, v}(A) \oplus \check{H}_{k}^{u, v}(B)=\operatorname{im} g_{k}$, and $\check{H}_{k}^{u, v}(X)=\operatorname{im} h_{k}$, where $f_{k}, g_{k}, h_{k}$ are as in diagram (2).

We begin by showing that $\beta$ is surjective. Let $c \in \operatorname{im} h_{k}$. There exists $d \in \check{H}_{k}\left(X_{u}\right)$ such that $h_{k}(d)=c$. By the exactness of the first horizontal line in diagram (2), the assumption rkker $\alpha_{k-1}^{u}=0$ implies that rkim $\delta_{k-1}^{u}=$ $\operatorname{rk} \breve{H}_{k}\left(X_{u}\right)-\operatorname{rkim} \beta_{k}^{u}=0$, i.e. that $\beta_{k}^{u}$ is surjective. Then there exists $d^{\prime} \in \check{H}_{k}\left(A_{u}\right) \oplus \check{H}_{k}\left(B_{u}\right)$ such that $h_{k} \circ \beta_{k}^{u}\left(d^{\prime}\right)=c$. By Lemma 3.2, $\beta_{k}^{v} \circ g_{k}\left(d^{\prime}\right)=c$. Thus, taking $c^{\prime}=g_{k}\left(d^{\prime}\right)$, we immediately have $\beta\left(c^{\prime}\right)=c$.

As for the injectivity of $\alpha$, the claim is immediate because $\operatorname{ker} \alpha \subseteq \operatorname{ker} \alpha_{k}^{v}$ and we are assuming $\alpha_{k}^{v}$ injective.

Now we have to show that $\operatorname{rkim} \alpha=\operatorname{rk} \operatorname{ker} \beta$. In order to do so, we observe that, if $\alpha_{k-1}^{u}$ is injective, and, consequently, $\beta$ is surjective, then

$$
\operatorname{rk} \check{H}_{k}^{u, v}(X)=\operatorname{rkim} \beta=\operatorname{rk} H_{k}^{u, v}(A) \oplus \check{H}_{k}^{u, v}(B)-\operatorname{rkker} \beta
$$

On the other hand, by Corollary 3.8, when $\operatorname{rkker} \alpha_{k}^{v}-\operatorname{rkker} \alpha_{k}^{v, u}+\operatorname{rk} \operatorname{ker} \alpha_{k-1}^{u}=0$ it holds that

$$
\operatorname{rk} \check{H}_{k}^{u, v}(X)=\operatorname{rk} \check{H}_{k}^{u, v}(A)+\operatorname{rk} \check{H}_{k}^{u, v}(B)-\operatorname{rk} \check{H}_{k}^{u, v}(A \cap B)
$$

Hence, if $\operatorname{rkker} \alpha_{k-1}^{u}=0$ and $\operatorname{rkker} \alpha_{k}^{v}=\operatorname{rkker} \alpha_{k}^{v, u}$, then $\operatorname{rk} \operatorname{ker} \beta=\operatorname{rk} \check{H}_{k}^{u, v}(A \cap B)$. Moreover, $\operatorname{since} \operatorname{rk} \check{H}_{k}^{u, v}(A \cap$ $B)=\operatorname{rkker} \alpha+\operatorname{rkim} \alpha$, when $\operatorname{rkker} \alpha_{k-1}^{u}=0$ and $\operatorname{rkker} \alpha_{k}^{v}=\operatorname{rkker} \alpha_{k}^{v, u}$ we have $\operatorname{rk} \operatorname{ker} \beta=\operatorname{rk} \operatorname{ker} \alpha+\operatorname{rkim} \alpha$. Therefore, when rk ker $\alpha_{k}^{v}=\operatorname{rkker} \alpha_{k}^{v, u}=\operatorname{rkker} \alpha_{k-1}^{u}=0, \alpha$ is injective if and only if $\operatorname{rkim} \alpha=\operatorname{rk} \operatorname{ker} \beta$.

\subsection{Examples}

In this subsection, we give two examples illustrating the previous results for the case $k=0$.

In both these examples, we consider a "bone" shaped object $A$, partially occluded by another object $B$, resulting in different shapes $X=A \cup B \subset \mathbb{R}^{2}$. The ranks of $\check{H}_{0}^{u, v}(A), \check{H}_{0}^{u, v}(B), \check{H}_{0}^{u, v}(A \cap B), \check{H}_{0}^{u, v}(X)$ are computed taking $\varphi: X \rightarrow \mathbb{R}, \varphi(P)=-\|P-H\|$, with $H$ a fixed point in $\mathbb{R}^{2}$.

In the first example, shown in Figure 3, the relation $\operatorname{rk} \check{H}_{0}^{u, v}(X)=\operatorname{rk} \check{H}_{0}^{u, v}(A)+\operatorname{rk} \check{H}_{0}^{u, v}(B)-\operatorname{rk} \check{H}_{0}^{u, v}(A \cap B)$, given in Corollary 3.8, holds for every proper point $(u, v) \in \Delta^{*}$.

In the second example, shown in Figure 4, the foreground object $B$ occludes $A$ in such a way that the relation given in Corollary 3.8 results not valid everywhere in $\Delta^{*}$, in accordance with Corollary 3.8. More precisely, rkker $\alpha_{0}^{v}=0$ for $v<-g$, while rkker $\alpha_{0}^{v}=1$ for $v \geq-g$. As for $\operatorname{ker} \alpha_{0}^{v, u}$, it has the same rank as ker $\alpha_{0}^{v}$ for every $u<v$, except when $-d \leq u<-e$ and $-e \leq v<-g$, because, in that case, rk ker $\alpha_{0}^{v, u}=1$, and, moreover, when $-e \leq u$ and $-g \leq v$, because, in that case, rk ker $\alpha_{0}^{v, u}=0$. To simplify the visualization of the points of $\Delta^{*}$ at which the equality $\operatorname{rk} \breve{H}_{0}^{u, v}(X)=\operatorname{rk} \breve{H}_{0}^{u, v}(A)+\operatorname{rk} \breve{H}_{0}^{u, v}(B)-\operatorname{rk} \breve{H}_{0}^{u, v}(A \cap B)$ does not hold, we refer the reader to Figure $4(\mathrm{c})$, where regions that do not verify such a relation are underlined by coloring them.

\section{The persistence diagram of the union of two spaces}

As recalled in Section 2, in earlier papers [5, 12, 3], it was shown that persistent homology groups can be concisely represented by collections of points with multiplicities, called persistence diagrams. 


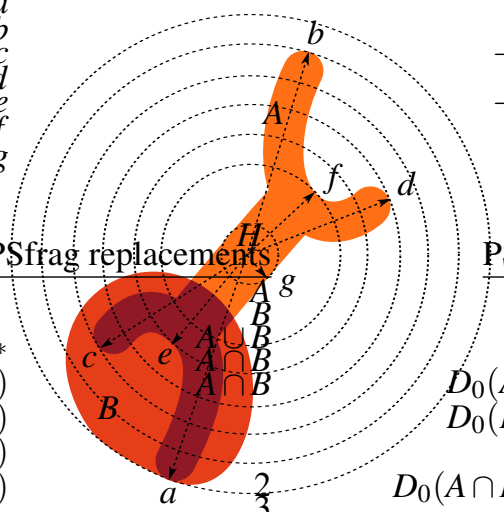

(a)

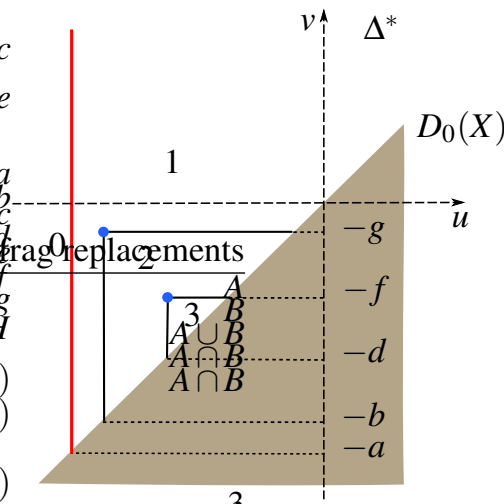

(b)

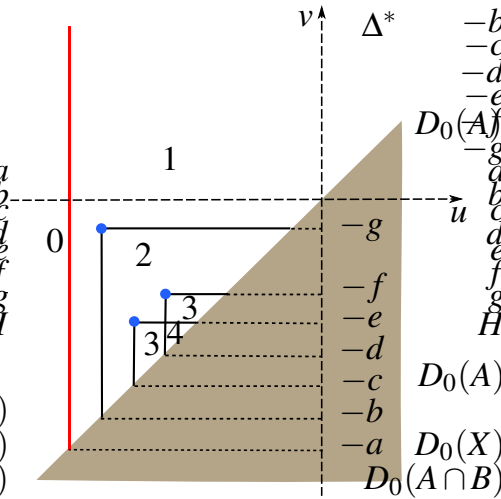

(c)

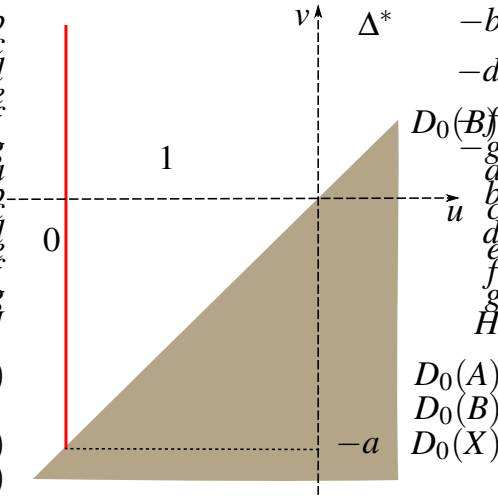

(d)

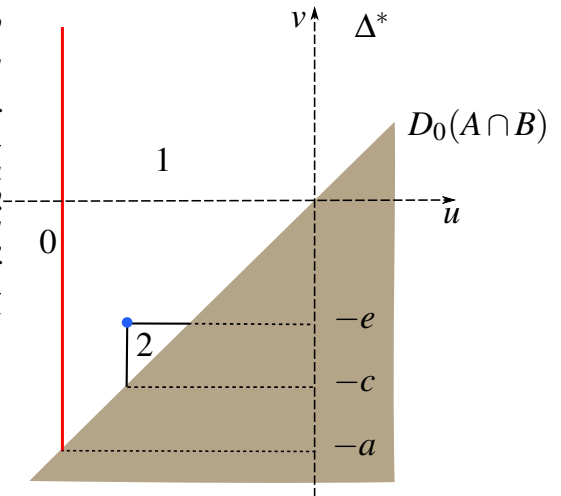

(e)

Figure 3: In (a) a "bone" shaped object $A$ is occluded by another object $B$. In (b), (c), (d) and (e) we show the points of $D_{0}(X, \varphi)$, $D_{0}\left(A, \varphi_{\mid A}\right), D_{0}\left(B, \varphi_{\mid B}\right)$, and $D_{0}\left(A \cap B, \varphi_{\mid A \cap B}\right)$, respectively, computed taking $\varphi: X \rightarrow \mathbb{R}, \varphi(P)=-\|P-H\|$, and the values of the 0th persistent homology group rank in each region of $\Delta^{*}$. In this example the relation $\operatorname{rk} \breve{H}_{0}^{u, v}(X)=\operatorname{rk} \breve{H}_{0}^{u, v}(A)+\operatorname{rk} \breve{H}_{0}^{u, v}(B)-\operatorname{rk} \breve{H}_{0}^{u, v}(A \cap B)$ of Corollary 3.8 holds everywhere in $\Delta^{*}$. 


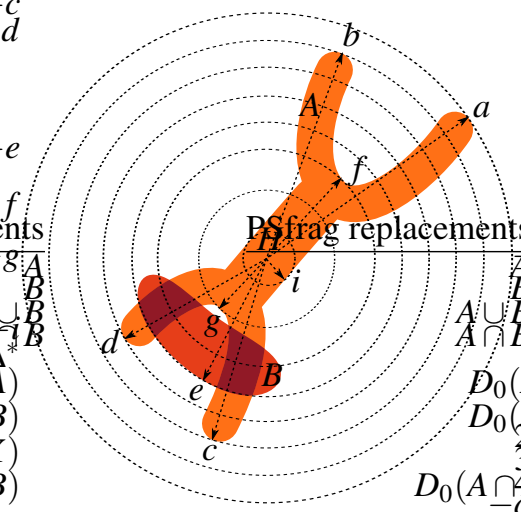

(a)

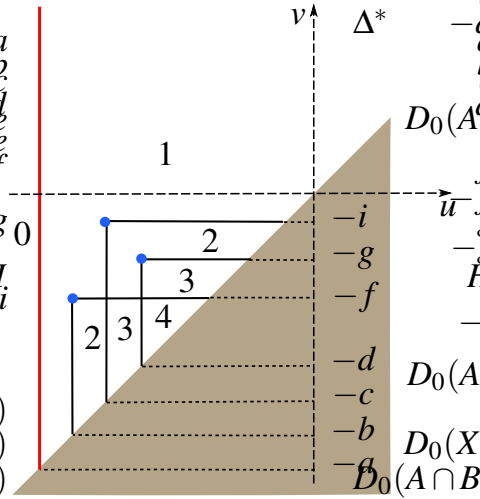

(d)

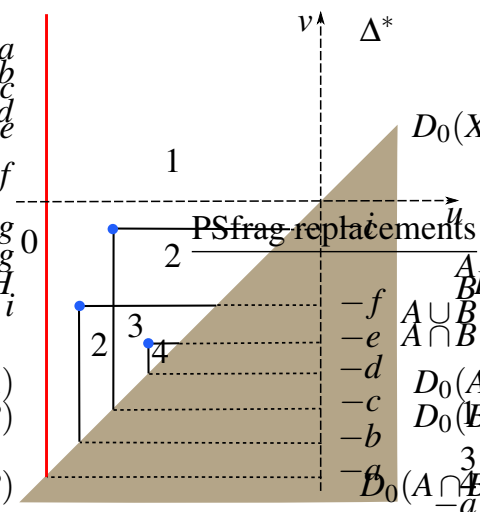

(b)

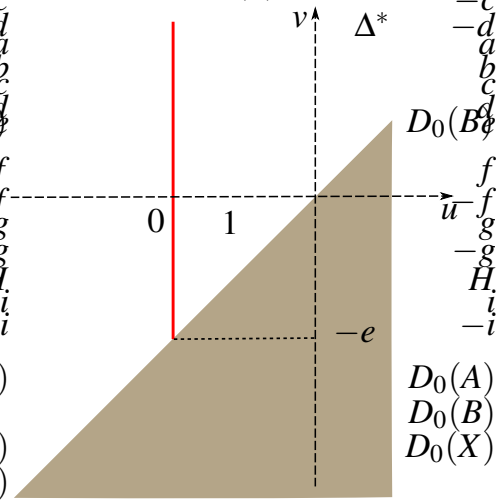

(e)

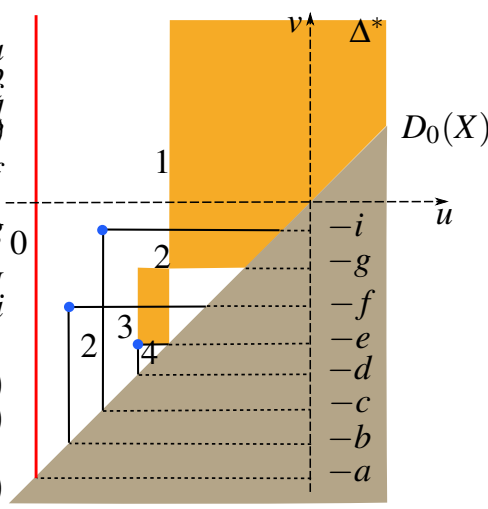

(c)

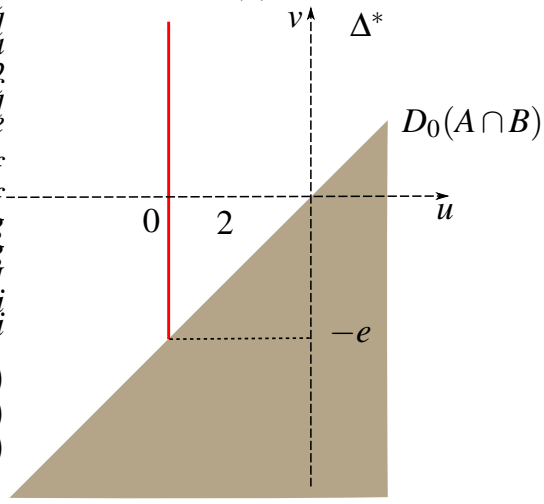

(f)

Figure 4: In (a) a "bone" shaped object $A$ is occluded by another object $B$. In (b), (d), (e), (f) we display the points of $D_{0}(X, \varphi), D_{0}\left(A, \varphi_{\mid A}\right)$, $D_{0}\left(B, \varphi_{\mid B}\right)$, and $D_{0}\left(A \cap B, \varphi_{\mid A \cap B}\right)$, respectively, computed taking $\varphi: X \rightarrow \mathbb{R}, \varphi(P)=-\|P-H\|$, and the values of the 0 th persistent homology group rank in each region of $\Delta^{*}$. In this case the relation $\operatorname{rk} \breve{H}_{0}^{u, v}(X)=\operatorname{rk} \breve{H}_{0}^{u, v}(A)+\operatorname{rk} \breve{H}_{0}^{u, v}(B)-\operatorname{rk} \breve{H}_{0}^{u, v}(A \cap B)$ of Corollary 3.8 does not hold everywhere in $\Delta^{*}$. In (c) we underline the regions of $\Delta^{*}$ where the equality is not valid by coloring them. 
This representation has the important property of being stable against continuous deformations of the space (or, which is equivalent, perturbations of the filtering functions). For this reason, in dealing with the shape comparison problem, via persistent homology groups, one actually compares persistence diagrams using either the Hausdorff distance or the matching (or bottleneck) distance (see, e.g., [5, 7, 6, 18]). The Hausdorff distance and the matching distance differ in that the former does not take into account multiplicities, while the latter does.

The aim of this section is to show the properties of the persistence diagram of the union of two spaces. Under the usual assumption that $X=A \cup B$, we prove that each point of the persistence diagram of $X$ is a point of the persistence diagram of $A$ or $B$, provided that one condition holds (Corollary 4.2). Moreover, under a finiteness condition, points of $D_{k}(A)$ and $D_{k}(B)$ either survive in $D_{k}(X)$ or in $D_{k}(A \cap B)$ (Theorem 4.3). However, in the particular case $k=0$, it always holds that the coordinates of points of $D_{0}(X)$ are also coordinates of points of $D_{0}(A)$ or $D_{0}(B)$ or $D_{0}(A \cap B)$ (Theorems 4.4 and 4.5).

These facts suggest that in persistent homology the partial matching of an occluded shape with the original shape can be translated into the partial matching of points of the corresponding persistence diagrams. This intuition will be developed in the experimental Section 5 for the special case $k=0$.

In the next proposition we obtain a relation involving the multiplicities of points of persistence diagrams $D_{k}(X), D_{k}(A)$ and $D_{k}(B)$. The main tool is the Mayer-Vietoris formula given in Theorem 3.7.

Proposition 4.1. Let $k \in \mathbb{Z}$. Then the following statements hold:

(i) for every proper point $p=(\bar{u}, \bar{v}) \in \Delta^{*}$, it holds that

$$
\begin{aligned}
\mu_{X, k}(p)-\mu_{A, k}(p)-\mu_{B, k}(p)+\mu_{A \cap B, k}(p)= & \lim _{\varepsilon \rightarrow 0^{+}}\left(\operatorname{rkker} \alpha_{k}^{\bar{v}-\varepsilon, \bar{u}-\varepsilon}-\operatorname{rkker} \alpha_{k}^{\overline{\bar{v}}-\varepsilon, \bar{u}+\varepsilon}\right. \\
& \left.+\operatorname{rkker} \alpha_{k}^{\overline{\bar{v}}+\varepsilon, \bar{u}+\varepsilon}-\operatorname{rkker} \alpha_{k}^{\overline{\bar{v}}+\varepsilon, \bar{u}-\varepsilon}\right) .
\end{aligned}
$$

(ii) for every point at infinity $r=(\bar{u}, \infty) \in \Delta^{*}$, it holds that

$$
\begin{array}{r}
\mu_{X, k}(r)-\mu_{A, k}(r)-\mu_{B, k}(r)+\mu_{A \cap B, k}(r)=\lim _{\varepsilon \rightarrow 0^{+}}\left(\operatorname{rkker} \alpha_{k}^{1 / \varepsilon, \bar{u}-\varepsilon}-\operatorname{rkker} \alpha_{k}^{1 / \varepsilon, \bar{u}+\varepsilon}\right. \\
\left.+\operatorname{rkker} \alpha_{k-1}^{\bar{u}+\varepsilon}-\operatorname{rkker} \alpha_{k-1}^{\bar{u}-\varepsilon}\right) .
\end{array}
$$

Proof. Let us prove only statement $(i)$, since $(i i)$ can be analogously verified.

Applying Theorem 3.7 four times with $(u, v)=(\bar{u}+\varepsilon, \bar{v}-\varepsilon),(u, v)=(\bar{u}-\varepsilon, \bar{v}-\varepsilon),(u, v)=(\bar{u}+\varepsilon, \bar{v}+\varepsilon)$, $(u, v)=(\bar{u}-\varepsilon, \bar{v}+\varepsilon), \varepsilon$ being a positive real number so small that $\bar{u}+\varepsilon<\bar{v}-\varepsilon$, we get

$$
\begin{aligned}
& \operatorname{rk} \check{H}_{k}^{\bar{u}+\varepsilon, \bar{v}-\varepsilon}(X)-\operatorname{rk} \check{H}_{k}^{\bar{u}-\varepsilon, \bar{v}-\varepsilon}(X)-\operatorname{rk} \breve{H}_{k}^{\bar{u}+\varepsilon, \bar{v}+\varepsilon}(X)+\operatorname{rk} \breve{H}_{k}^{\bar{u}-\varepsilon, \bar{v}+\varepsilon}(X) \\
& =\operatorname{rk} \breve{H}_{k}^{\bar{u}+\varepsilon, \bar{v}-\varepsilon}(A)+\operatorname{rk} \breve{H}_{k}^{\bar{u}+\varepsilon, \bar{v}-\varepsilon}(B)-\operatorname{rk} \breve{H}_{k}^{\bar{u}+\varepsilon, \bar{v}-\varepsilon}(A \cap B)+\operatorname{rkker} \alpha_{k}^{\bar{v}-\varepsilon}-\operatorname{rk} \operatorname{ker} \alpha_{k}^{\bar{v}-\varepsilon, \bar{u}+\varepsilon}+\operatorname{rk} \operatorname{ker} \alpha_{k-1}^{\bar{u}+\varepsilon} \\
& -\left(\operatorname{rk} \check{H}_{k}^{\bar{u}-\varepsilon, \bar{v}-\varepsilon}(A)+\operatorname{rk} \check{H}_{k}^{\bar{u}-\varepsilon, \bar{v}-\varepsilon}(B)-\operatorname{rk} \breve{H}_{k}^{\bar{u}-\varepsilon, \bar{v}-\varepsilon}(A \cap B)+\operatorname{rkker} \alpha_{k}^{\bar{v}-\varepsilon}-\operatorname{rkker} \alpha_{k}^{\bar{v}-\varepsilon, \bar{u}-\varepsilon}+\operatorname{rkker} \alpha_{k-1}^{\bar{u}-\varepsilon}\right) \\
& -\left(\operatorname{rk} \check{H}_{k}^{\bar{u}+\varepsilon, \bar{v}+\varepsilon}(A)+\operatorname{rk} \check{H}_{k}^{\bar{u}+\varepsilon, \bar{v}+\varepsilon}(B)-\operatorname{rk} \check{H}_{k}^{\bar{u}+\varepsilon, \bar{v}+\varepsilon}(A \cap B)+\operatorname{rkker} \alpha_{k}^{\bar{v}+\varepsilon}-\operatorname{rk} \operatorname{ker} \alpha_{k}^{\bar{v}+\varepsilon, \bar{u}+\varepsilon}+\operatorname{rkker} \alpha_{k-1}^{\bar{u}+\varepsilon}\right) \\
& +\operatorname{rk} \breve{H}_{k}^{\bar{u}-\varepsilon, \bar{v}+\varepsilon}(A)+\operatorname{rk} \check{H}_{k}^{\bar{u}-\varepsilon, \bar{v}+\varepsilon}(B)-\operatorname{rk} \breve{H}_{k}^{\bar{u}-\varepsilon, \bar{v}+\varepsilon}(A \cap B)+\operatorname{rkker} \alpha_{k}^{\bar{v}+\varepsilon}-\operatorname{rkker} \alpha_{k}^{\bar{v}+\varepsilon, \bar{u}-\varepsilon}+\operatorname{rk} \operatorname{ker} \alpha_{k-1}^{\bar{u}-\varepsilon} \\
& =\operatorname{rk} \breve{H}_{k}^{\bar{u}+\varepsilon, \bar{v}-\varepsilon}(A)-\operatorname{rk} \breve{H}_{k}^{\bar{u}-\varepsilon, \bar{v}-\varepsilon}(A)-\operatorname{rk} \breve{H}_{k}^{\bar{u}+\varepsilon, \bar{v}+\varepsilon}(A)+\operatorname{rk} \breve{H}_{k}^{\bar{u}-\varepsilon, \bar{v}+\varepsilon}(A) \\
& +\operatorname{rk} \check{H}_{k}^{\bar{u}+\varepsilon, \bar{v}-\varepsilon}(B)-\operatorname{rk} \check{H}_{k}^{\bar{u}-\varepsilon, \bar{v}-\varepsilon}(B)-\operatorname{rk} \check{H}_{k}^{\bar{u}+\varepsilon, \bar{v}+\varepsilon}(B)+\operatorname{rk} \check{H}_{k}^{\bar{u}-\varepsilon, \bar{v}+\varepsilon}(B) \\
& -\left(\operatorname{rk}_{k}^{\bar{u}+\varepsilon, \bar{v}-\varepsilon}(A \cap B)-\operatorname{rk} \breve{H}_{k}^{\bar{u}-\varepsilon, \bar{v}-\varepsilon}(A \cap B)-\operatorname{rk} \breve{H}_{k}^{\bar{u}+\varepsilon, \bar{v}+\varepsilon}(A \cap B)+\operatorname{rk} \check{H}_{k}^{\bar{u}-\varepsilon, \bar{v}+\varepsilon}(A \cap B)\right) \\
& +\operatorname{rkker} \alpha_{k}^{\bar{v}-\varepsilon, \bar{u}-\varepsilon}-\operatorname{rkker} \alpha_{k}^{\bar{v}-\varepsilon, \bar{u}+\varepsilon}+\operatorname{rkker} \alpha_{k}^{\bar{v}+\varepsilon, \bar{u}+\varepsilon}-\operatorname{rkker} \alpha_{k}^{\bar{v}+\varepsilon, \bar{u}-\varepsilon} \text {. }
\end{aligned}
$$


Hence, by definition of multiplicity of a proper point of $\Delta^{*}$ (Definition 2.2), we have

$$
\begin{aligned}
\lim _{\varepsilon \rightarrow 0^{+}} & \left(\operatorname{rk} \operatorname{ker} \alpha_{k}^{\bar{v}-\varepsilon, \bar{u}-\varepsilon}-\operatorname{rkker} \alpha_{k}^{\bar{v}-\varepsilon, \bar{u}+\varepsilon}+\operatorname{rkker} \alpha_{k}^{\bar{v}+\varepsilon, \bar{u}+\varepsilon}-\operatorname{rkker} \alpha_{k}^{\bar{v}+\varepsilon, \bar{u}-\varepsilon}\right) \\
= & \lim _{\varepsilon \rightarrow 0^{+}}\left(\operatorname{rk} \breve{H}_{k}^{\bar{u}+\varepsilon, \bar{v}-\varepsilon}(X)-\operatorname{rk} \breve{H}_{k}^{\bar{u}-\varepsilon, \bar{v}-\varepsilon}(X)-\operatorname{rk} \breve{H}_{k}^{\bar{u}+\varepsilon, \bar{v}+\varepsilon}(X)+\operatorname{rk} \breve{H}_{k}^{\bar{u}-\varepsilon, \bar{v}+\varepsilon}(X)\right) \\
& -\lim _{\varepsilon \rightarrow 0^{+}}\left(\operatorname{rk} \check{H}_{k}^{\bar{u}+\varepsilon, \bar{v}-\varepsilon}(A)-\operatorname{rk} \breve{H}_{k}^{\bar{u}-\varepsilon, \bar{v}-\varepsilon}(A)-\operatorname{rk} \check{H}_{k}^{\bar{u}+\varepsilon, \bar{v}+\varepsilon}(A)+\operatorname{rk} \breve{H}_{k}^{\bar{u}-\varepsilon, \bar{v}+\varepsilon}(A)\right) \\
& -\lim _{\varepsilon \rightarrow 0^{+}}\left(\operatorname{rk} \check{H}_{k}^{\bar{u}+\varepsilon, \bar{v}-\varepsilon}(B)-\operatorname{rk} \breve{H}_{k}^{\bar{u}-\varepsilon, \bar{v}-\varepsilon}(B)-\operatorname{rk} \check{H}_{k}^{\bar{u}+\varepsilon, \bar{v}+\varepsilon}(B)+\operatorname{rk} \breve{H}_{k}^{\bar{u}-\varepsilon, \bar{v}+\varepsilon}(B)\right) \\
& +\lim _{\varepsilon \rightarrow 0^{+}}\left(\operatorname{rk} \check{H}_{k}^{\bar{u}+\varepsilon, \bar{v}-\varepsilon}(A \cap B)-\operatorname{rk} \breve{H}_{k}^{\bar{u}-\varepsilon, \bar{v}-\varepsilon}(A \cap B)-\operatorname{rk} \breve{H}_{k}^{\bar{u}+\varepsilon, \bar{v}+\varepsilon}(A \cap B)+\operatorname{rk} \check{H}_{k}^{\bar{u}-\varepsilon, \bar{v}+\varepsilon}(A \cap B)\right) \\
= & \mu_{X, k}(p)-\mu_{A, k}(p)-\mu_{B, k}(p)+\mu_{A \cap B, k}(p) .
\end{aligned}
$$

Using the previous Proposition 4.1, and recalling that points of a persistence diagram are those with a positive multiplicity, we find a condition ensuring that both points of $D_{k}(X)$ and points of $D_{k}(A \cap B)$ are also points of $D_{k}(A)$ or $D_{k}(B)$.

Corollary 4.2. For every $k \in \mathbb{Z}$, the following statements hold:

- If the proper point $p=(\bar{u}, \bar{v}) \in \Delta^{*}$ belongs to $D_{k}(X) \cup D_{k}(A \cap B)$ and

$$
\lim _{\varepsilon \rightarrow 0^{+}}\left(\operatorname{rkker} \alpha_{k}^{\bar{v}-\varepsilon, \bar{u}-\varepsilon}-\operatorname{rk} \operatorname{ker} \alpha_{k}^{\bar{v}-\varepsilon, \bar{u}+\varepsilon}+\operatorname{rk} \operatorname{ker} \alpha_{k}^{\bar{v}+\varepsilon, \bar{u}+\varepsilon}-\operatorname{rkker} \alpha_{k}^{\bar{v}+\varepsilon, \bar{u}-\varepsilon}\right) \leq 0,
$$

then $p$ belongs to $D_{k}(A) \cup D_{k}(B)$.

- If the point at infinity $r=(\bar{u}, \infty) \in \Delta^{*}$ belongs to $D_{k}(X) \cup D_{k}(A \cap B)$ and

$$
\lim _{\varepsilon \rightarrow 0^{+}}\left(\operatorname{rkker} \alpha_{k}^{1 / \varepsilon, \bar{u}-\varepsilon}-\operatorname{rkker} \alpha_{k}^{1 / \varepsilon, \bar{u}+\varepsilon}+\operatorname{rkker} \alpha_{k-1}^{\bar{u}+\varepsilon}-\operatorname{rkker} \alpha_{k-1}^{\bar{u}-\varepsilon}\right) \leq 0
$$

then $r$ belongs to $D_{k}(A) \cup D_{k}(B)$.

Proof. Let us prove only the first statement, since the second one can be seen similarly.

Let $\lim _{\varepsilon \rightarrow 0^{+}}\left(\operatorname{rkker} \alpha_{k}^{\bar{v}-\varepsilon, \bar{u}-\varepsilon}-\operatorname{rk} \operatorname{ker} \alpha_{k}^{\bar{v}-\varepsilon, \bar{u}+\varepsilon}+\operatorname{rk} \operatorname{ker} \alpha_{k}^{\bar{v}+\varepsilon, \bar{u}+\varepsilon}-\operatorname{rk} \operatorname{ker} \alpha_{k}^{\bar{v}+\varepsilon, \bar{u}-\varepsilon}\right) \leq 0$. From Proposition 4.1(i), we deduce that $\mu_{X, k}(p)+\mu_{A \cap B, k}(p) \leq \mu_{A, k}(p)+\mu_{B, k}(p)$. Since $p \in D_{k}(X) \cup D_{k}(A \cap B)$, that is $\mu_{X, k}(p)+$ $\mu_{A \cap B, k}(p)>0$, this immediately implies that either $\mu_{A, k}(p)>0$ or $\mu_{B, k}(p)>0$, proving the statement.

The following Theorem 4.3 is a sort of converse of Corollary 4.2. Although it is valid under restrictive conditions on the number of homological critical values of the filtering functions as a consequence of the results of Appendix B, it suggests that shape features of $A$ and $B$ either survive as points of $D_{k}(X)$ or can be found in $D_{k}(A \cap B)$.

Theorem 4.3. Let $k \in \mathbb{Z}$, and let us assume that both $\varphi$ and $\varphi_{\mid A \cap B}$ have at most a finite number of homological $k$-critical values. The following statements hold:

- Let $p=(\bar{u}, \bar{v}) \in \Delta^{*}$ be a proper point such that at least one of its coordinates is neither a homological $(k+1)$-critical value of $\varphi$ nor a homological $(k-1)$-critical value of $\varphi_{\mid A \cap B}$. If $p \in D_{k}(A) \cup D_{k}(B)$, then $p \in D_{k}(X) \cup D_{k}(A \cap B)$.

- Let $r=(\bar{u}, \infty) \in \Delta^{*}$ be a point at infinity such that $\bar{u}$ is neither a homological $(k+1)$-critical value of $\varphi$ nor a homological $(k-1)$-critical value of $\varphi_{\mid A \cap B}$. If $r \in D_{k}(A) \cup D_{k}(B)$, then $r \in D_{k}(X) \cup D_{k}(A \cap B)$. 
Proof. Let us prove only the first statement since the proof of the second one is similar.

By contradiction, let us assume that $p \notin D_{k}(X) \cup D_{k}(A \cap B)$. Let us consider the case when its abscissa $\bar{u}$ is neither a homological $(k+1)$-critical value of $\varphi$ nor a homological $(k-1)$-critical value of $\varphi_{\mid A \cap B}$. The proof for $\bar{v}$ is analogous. Since we are assuming that both $\varphi$ and $\varphi_{\mid A \cap B}$ have at most a finite number of homological $k$-critical values, the assumption that $p \notin D_{k}(X) \cup D_{k}(A \cap B)$ implies, by Proposition B.4, that $\bar{u}$ is a homological $k$-critical value for neither $\varphi$ nor $\varphi_{\mid A \cap B}$. Let us consider now the following commutative diagram

$$
\begin{gathered}
\check{H}_{k+1}\left(X_{\bar{v}-\varepsilon}, X_{\bar{u}-\varepsilon}\right) \stackrel{\delta_{k}^{\bar{v}-\varepsilon, \bar{u}-\varepsilon}}{\longrightarrow} \check{H}_{k}\left((A \cap B)_{\bar{v}-\varepsilon},(A \cap B)_{\bar{u}-\varepsilon}\right) \\
\downarrow_{i_{1}} \quad \downarrow_{i_{2}} \\
\check{H}_{k+1}\left(X_{\bar{v}-\varepsilon}, X_{\bar{u}+\varepsilon}\right) \stackrel{\delta_{k}^{\bar{v}-\varepsilon, \bar{u}+\varepsilon}}{\longrightarrow} \check{H}_{k}\left((A \cap B)_{\bar{v}-\varepsilon},(A \cap B)_{\bar{u}+\varepsilon}\right)
\end{gathered}
$$

for every sufficiently small $\varepsilon>0$, such that $\bar{u}+\varepsilon<\bar{v}-\varepsilon$, and let us show that, under our assumptions, the vertical maps $i_{1}$ and $i_{2}$, induced by inclusions, are isomorphisms. To see that $i_{1}$ is an isomorphism, it is sufficient to consider the following commutative diagram

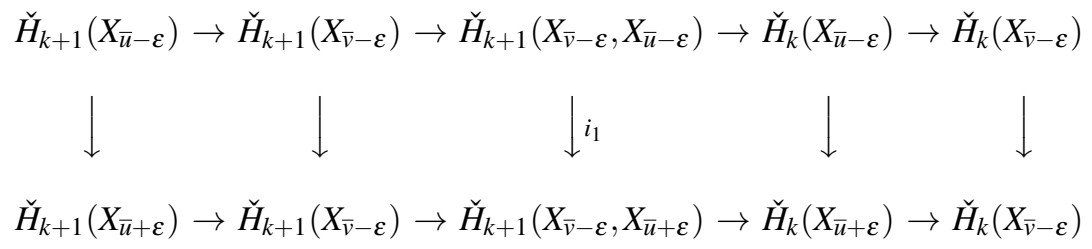

and apply the Five Lemma, recalling that $\bar{u}$ is neither a homological $(k+1)$-critical nor a homological $k$-critical value of $\varphi$.

Analogously, by recalling that $\bar{u}$ is neither a homological $k$-critical nor a homological $(k-1)$-critical value of $\varphi_{\mid A \cap B}$, and applying the Five Lemma to the following commutative diagram

$$
\begin{aligned}
\check{H}_{k}\left((A \cap B)_{\bar{u}-\varepsilon}\right) \rightarrow \check{H}_{k}\left((A \cap B)_{\bar{v}-\varepsilon}\right) & \rightarrow \check{H}_{k}\left((A \cap B)_{\bar{v}-\varepsilon},(A \cap B)_{\bar{u}-\varepsilon}\right) \rightarrow \check{H}_{k-1}\left((A \cap B)_{\bar{u}-\varepsilon}\right) \rightarrow \check{H}_{k-1}\left((A \cap B)_{\bar{v}-\varepsilon}\right) \\
\downarrow & \downarrow \\
\downarrow & \downarrow i_{2}
\end{aligned}
$$

we can deduce that $i_{2}$ is an isomorphism.

Therefore, from diagram (10), we get $\operatorname{rkim} \delta_{k}^{\bar{v}-\varepsilon, \bar{u}-\varepsilon}=\operatorname{rkim} \delta_{k}^{\bar{v}-\varepsilon, \bar{u}+\varepsilon}$ for every sufficiently small $\varepsilon>0$, or, equivalently, by Lemma 3.2, $\operatorname{rk} \operatorname{ker} \alpha_{k}^{\bar{v}-\varepsilon, \bar{u}-\varepsilon}=\operatorname{rk} \operatorname{ker} \alpha_{k}^{\bar{\nu}-\varepsilon, \bar{u}+\varepsilon}$ for every sufficiently small $\varepsilon>0$. By replacing $\bar{v}-\varepsilon$ with $\bar{v}+\varepsilon$ in the above diagram, it can also be proved that $\operatorname{rk} \operatorname{ker} \alpha_{k}^{\bar{v}+\varepsilon, \bar{u}-\varepsilon}=\operatorname{rkker} \alpha_{k}^{\bar{v}+\varepsilon, \bar{u}+\varepsilon}$ for every sufficiently small $\varepsilon>0$. Hence, by Proposition $4.1, \mu_{X, k}(p)-\mu_{A, k}(p)-\mu_{B, k}(p)+\mu_{A \cap B, k}(p)=0$. Since we are assuming that $\mu_{A \cap B, k}(p)=\mu_{X, k}(p)=0$, and $\mu_{A, 0}(p)+\mu_{B, 0}(p)>0$, we obtain a contradiction.

\subsection{Some results about points of the persistence diagram in the case $\boldsymbol{k}=0$}

In the case $k=0$ it is possible to prove further results linking the coordinates of the points of the persistence diagrams of $X, A, B$, and $A \cap B$. Precisely, the following two theorems state that the abscissas of points of $D_{0}(X)$ are abscissas of points of $D_{0}(A) \cup D_{0}(B) \cup D_{0}(A \cap B)$ (Theorem 4.4); the ordinates of the proper points of $D_{0}(X)$ are, in general, homological 0-critical values of either $\varphi_{\mid A}$ or $\varphi_{\mid B}$ or $\varphi_{\mid A \cap B}$, and, under finiteness conditions, abscissas or ordinates of points of $D_{0}(A) \cup D_{0}(B) \cup D_{0}(A \cap B)$ (Theorem 4.5). 
These facts can easily be seen in the examples illustrated in Figures 3-4. In particular, in Figure 4, the persistence diagram $D_{0}(X)$ presents the proper point $(-d,-e)$, which is not a point of $D_{0}(A) \cup D_{0}(B) \cup D_{0}(A \cap B)$. Nevertheless, its abscissa $-d$ is the abscissa of one of the points of $D_{0}(A)$, while its ordinate $-e$ is the abscissa of the sole point at infinity of both $D_{0}(B)$ and $D_{0}(A \cap B)$.

We point out that analogous statements for $k>0$ would be false.

Theorem 4.4. Let $p=(\bar{u}, \bar{v}) \in \Delta^{*}$ be a proper point. If $p \in D_{0}(X)$, then there exists $v>\bar{u}$ such that $(\bar{u}, v) \in$ $D_{0}(A) \cup D_{0}(B) \cup D_{0}(A \cap B)$. Moreover, let $r=(\bar{u}, \infty) \in \Delta^{*}$ be a point at infinity. If $r \in D_{0}(X)$, then $r \in D_{0}(A) \cup$ $D_{0}(B)$.

Proof. As for the first assertion, we proceed by contradiction.

Let us recall that the rank of persistent homology groups is non-decreasing with respect to the first parameter (Lemma B.2), so that $\operatorname{rk} \breve{H}_{0}^{\bar{u}+\varepsilon, \bar{v} \pm \varepsilon}(A \cap B) \geq \operatorname{rk} \check{H}_{0}^{\bar{u}-\varepsilon, \bar{v} \pm \varepsilon}(A \cap B), \operatorname{rk} \breve{H}_{0}^{\bar{u}+\varepsilon, \bar{v} \pm \varepsilon}(A) \geq \operatorname{rk} \breve{H}_{0}^{\bar{u}-\varepsilon, \bar{v} \pm \varepsilon}(A), \operatorname{rk} \breve{H}_{0}^{\bar{u}+\varepsilon, \bar{v} \pm \varepsilon}(B) \geq$ $\operatorname{rk} \check{H}_{0}^{\bar{u}-\varepsilon, \bar{v} \pm \varepsilon}(B)$.

Let us assume that for every $v>\bar{u}, \mu_{A, 0}(\bar{u}, v)=0, \mu_{B, 0}(\bar{u}, v)=0$ and $\mu_{A \cap B, 0}(\bar{u}, v)=0$. This implies that $\bar{u}$ is not the abscissa of a point of either $D_{0}(A), D_{0}(B)$, or $D_{0}(A \cap B)$. Then, by Lemma B.3 $(a)$, for every sufficiently small $\varepsilon$, the following equalities hold: $\operatorname{rk} \check{H}_{0}^{\bar{u}+\varepsilon, \bar{v} \pm \varepsilon}(A \cap B)=\operatorname{rk} \check{H}_{0}^{\bar{u}-\varepsilon, \bar{v} \pm \varepsilon}(A \cap B), \operatorname{rk} \check{H}_{0}^{\bar{u}+\varepsilon, \bar{v} \pm \varepsilon}(A)=\operatorname{rk} \breve{H}_{0}^{\bar{u}-\varepsilon, \bar{v} \pm \varepsilon}(A)$, $\operatorname{rk} \breve{H}_{0}^{\bar{u}+\varepsilon, \bar{v} \pm \varepsilon}(B)=\operatorname{rk}_{0}^{\bar{u}-\varepsilon, \bar{v} \pm \varepsilon}(B)$.

Now, from Lemma 3.6, $\operatorname{rk} \breve{H}_{0}^{\bar{u}+\varepsilon, \bar{v}-\varepsilon}(A \cap B)=\operatorname{rk} \breve{H}_{0}^{\bar{u}-\varepsilon, \bar{v}-\varepsilon}(A \cap B)$ implies that $\operatorname{rk} \check{H}_{0}\left((A \cap B)_{\bar{v}-\varepsilon},(A \cap B)_{\bar{u}+\varepsilon}\right)=$ $\operatorname{rk} \check{H}_{0}\left((A \cap B)_{\bar{v}-\varepsilon},(A \cap B)_{\bar{u}-\varepsilon}\right)$. Similarly, we get $\operatorname{rk} \check{H}_{0}\left(A_{\bar{v}-\varepsilon}, A_{\bar{u}+\varepsilon}\right)=\operatorname{rk} \check{H}_{0}\left(A_{\bar{v}-\varepsilon}, A_{\bar{u}-\varepsilon}\right)$ and $\operatorname{rk} \check{H}_{0}\left(B_{\bar{v}-\varepsilon}, B_{\bar{u}+\varepsilon}\right)=$ $\operatorname{rk} \breve{H}_{0}\left(B_{\bar{v}-\varepsilon}, B_{\bar{u}-\varepsilon}\right)$.

Next, let us consider the following commutative diagram

$$
\begin{gathered}
\check{H}_{0}\left((A \cap B)_{\bar{v}-\varepsilon},(A \cap B)_{\bar{u}-\varepsilon}\right) \stackrel{\alpha_{0}^{\bar{v}-\varepsilon, \bar{u}-\varepsilon}}{\longrightarrow} \check{H}_{0}\left(A_{\bar{v}-\varepsilon}, A_{\bar{u}-\varepsilon}\right) \oplus \check{H}_{0}\left(B_{\bar{v}-\varepsilon}, B_{\bar{u}-\varepsilon}\right) \\
\downarrow_{j_{1}} \downarrow j_{2} \\
\check{H}_{0}\left((A \cap B)_{\bar{v}-\varepsilon},(A \cap B)_{\bar{u}+\varepsilon}\right) \stackrel{\alpha_{0}^{\overline{\bar{v}}-\varepsilon, \bar{u}+\varepsilon}}{\longrightarrow} \check{H}_{0}\left(A_{\bar{v}-\varepsilon}, A_{\bar{u}+\varepsilon}\right) \oplus \check{H}_{0}\left(B_{\bar{v}-\varepsilon}, B_{\bar{u}+\varepsilon}\right)
\end{gathered}
$$

for any sufficiently small $\varepsilon>0$ such that $\bar{u}+\varepsilon<\bar{v}-\varepsilon$, and let us show that the vertical maps $j_{1}$ and $j_{2}$, induced by inclusions, are surjective. The surjectivity of $j_{1}$ easily follows chasing the commutative diagram below, whose horizontal lines are exact:

$$
\begin{gathered}
\check{H}_{0}\left((A \cap B)_{\bar{v}-\varepsilon}\right) \rightarrow \check{H}_{0}\left((A \cap B)_{\bar{v}-\varepsilon},(A \cap B)_{\bar{u}-\varepsilon}\right) \rightarrow 0 \\
\downarrow \downarrow j_{1} \\
\qquad \check{H}_{0}\left((A \cap B)_{\bar{v}-\varepsilon}\right) \rightarrow \check{H}_{0}\left((A \cap B)_{\bar{v}-\varepsilon},(A \cap B)_{\bar{u}+\varepsilon}\right) \rightarrow 0
\end{gathered}
$$

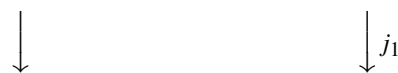

The surjectivity of $j_{2}$ can be seen analogously.

Since $j_{1}$ and $j_{2}$ are surjective and their respective domain and codomain have the same finite rank, we deduce that they are isomorphisms. So, from diagram (11), we obtain $\operatorname{ker} \alpha_{0}^{\bar{v}-\varepsilon, \bar{u}-\varepsilon} \simeq \operatorname{ker} \alpha_{0}^{\bar{v}-\varepsilon, \bar{u}+\varepsilon}$ for any sufficiently small $\varepsilon>0$.

Proceeding analogously, we can prove that $\operatorname{ker} \alpha_{0}^{\bar{v}+\varepsilon, \bar{u}-\varepsilon} \simeq \operatorname{ker} \alpha_{0}^{\bar{v}+\varepsilon, \bar{u}+\varepsilon}$ for any sufficiently small $\varepsilon>0$.

Therefore, applying Proposition 4.1, it follows that $\mu_{X, 0}(p)-\mu_{A, 0}(p)-\mu_{B, 0}(p)+\mu_{A \cap B, 0}(p)=0$. In particular, by the assumption that $\mu_{A, 0}(p)=0, \mu_{B, 0}(p)=0$ and $\mu_{A \cap B, 0}(p)=0$, it holds that $\mu_{X, 0}(p)=0$, a contradiction.

In the case of points at infinity, we observe that, if $(\bar{u}, \infty)$ belongs to $D_{0}(X)$, then $\bar{u}=\min _{P \in C} \varphi(P)$ for at least one connected component $C$ of $X$ ([12, Prop. 9]). Furthermore, since $X=A \cup B$, it follows that $\bar{u}=\min _{P \in C \cap A} \varphi_{\mid A}(P)$ 
or $\bar{u}=\min _{P \in C \cap B} \varphi_{\mid B}(P)$, from which (by [12, Prop. 9]), $(\bar{u}, \infty)$ is shown to be a point at infinity of either $D_{0}(A)$ or $D_{0}(B)$

Theorem 4.5. Let $p=(\bar{u}, \bar{v}) \in \Delta^{*}$ be a proper point. If $p \in D_{0}(X)$, then $\bar{v}$ is a homological 0 -critical value of $\varphi_{\mid A}$ or $\varphi_{\mid B}$ or $\varphi_{\mid A \cap B}$. Furthermore, if $\varphi_{\mid A}, \varphi_{\mid B}$, and $\varphi_{\mid A \cap B}$ have at most a finite number of homological 0critical values, then $\bar{v}$ is the abscissa of a point (proper or at infinity) or the ordinate of a proper point of $D_{0}(A) \cup D_{0}(B) \cup D_{0}(A \cap B)$.

Proof. Regarding the first assertion, we proceed by contradiction.

Let us assume that $\bar{v}$ is not a homological 0-critical value of $\varphi_{\mid A}, \varphi_{\mid B}$ and $\varphi_{\mid A \cap B}$. Then, by Definition B.1, for every $\bar{\varepsilon}>0$, there exists $\varepsilon$ with $0<\varepsilon<\bar{\varepsilon}$, such that the homomorphisms $f$ and $g$ induced by inclusions in the following commutative diagram with exact horizontal lines

$$
\begin{aligned}
& \check{H}_{0}\left((A \cap B)_{\bar{v}-\varepsilon}\right) \rightarrow \check{H}_{0}\left(A_{\bar{v}-\varepsilon}\right) \oplus \check{H}_{0}\left(B_{\bar{v}-\varepsilon}\right) \rightarrow \check{H}_{0}\left(X_{\bar{v}-\varepsilon}\right) \rightarrow 0 \\
& \downarrow f \quad \downarrow g \quad \downarrow \downarrow_{0}^{\bar{c}-\varepsilon, \bar{v}+\varepsilon} \quad \downarrow_{0} \\
& \check{H}_{0}\left((A \cap B)_{\bar{v}+\varepsilon}\right) \rightarrow \check{H}_{0}\left(A_{\bar{v}+\varepsilon}\right) \oplus \check{H}_{0}\left(B_{\bar{v}+\varepsilon}\right) \rightarrow \check{H}_{0}\left(X_{\bar{v}+\varepsilon}\right) \rightarrow 0
\end{aligned}
$$

are isomorphisms. Therefore, extending the horizontal lines of the above diagram rightwards with two trivial homomorphisms, we can apply the Five Lemma and deduce that $l_{0}^{\bar{v}-\varepsilon, \bar{v}+\varepsilon}$ is an isomorphism. This implies that $\bar{v}$ is not a homological 0-critical value of $\varphi$. Consequently, applying Proposition B.4, it holds that, for every $u<\bar{v}$, $\mu_{X, 0}(u, \bar{v})=0$, giving a contradiction.

As for the second statement, it is immediate consequence of the second statement of Proposition B.4.

\section{Shape recognition in the presence of occlusions}

In this section we apply the results of the previous sections concerning the relationship among points of the persistence diagrams of $X=A \cup B, A$ and $B$ to a problem of partial matching. In particular we focus on shape recognition under partial occlusions. We model the presence of occlusions in a shape as follows. The object of interest $A$ is occluded by a foreground object $B$, so that the visible object $X$ is given by $A \cup B$.

Psychophysical observations indicate that human and monkey perception of partially occluded shapes changes according to whether, or not, the occluding pattern is visible to the observer, and whether the occluded shape is a filled figure or an outline [17]. In particular, discrimination performance is higher for filled shapes than for outlines, and in both cases it significantly improves when shapes are occluded by a visible rather than invisible object.

In computer vision experiments, researcher usually work with invisible occluding patterns, both on outlines (see, e.g., [4, 13, 19, 21, 22]) and on filled shapes (see, e.g., [15]).

To test persistence diagram performance under occlusions, we work with 70 filled images, each chosen from a different class of the MPEG-7 dataset [20]. We carry out two experiments that differ in the visibility of the occluding pattern. In the first experiment the occluding pattern is visible, and we aim at finding a fingerprint of the original shape in the persistence diagram of the occluded shape. In the second experiment, where the occluding pattern is invisible, we perform a direct comparison between the occluded shape and the original shape. In both these experiments, the occluding pattern is a rectangular shape occluding from the top, or the left, by an area we increasingly vary from $20 \%$ to $60 \%$ of the height or width of the bounding box of the original shape. We compute persistence diagrams for both the original shapes and the occluded ones, choosing a family of eight filtrating functions having only the set of black pixels as domain. They are defined as follows: four of them as the distance from the line passing through the origin (top left point of the bounding box), rotated by an angle of $0, \frac{\pi}{4}, \frac{\pi}{2}$ and $\frac{3 \pi}{4}$ radians, respectively, with respect to the horizontal position; the other four as minus the 
distance from the same lines, respectively. This family of filtrating functions is chosen only for demonstrative purposes, since the associated persistence diagrams are simple in terms of the number of points, but, at the same time, non-trivial in terms of shape information. For dimensional reasons, we can confine computations to 0th degree persistent homology. We recall that the rank of 0th degree persistent homology groups was originally introduced under the name of size functions [11].

The first experiment aims to show how a trace of the persistence diagram describing the shape of an object is contained in the persistence diagram related to the occluded shape when the occluding pattern is visible (see first column of Tables 2-4). With reference to the notation used in our theoretical setting, we are considering $A$ as the original shape, $B$ as the black rectangle, and $X$ as the occluded shape generated by their union.

In Table 1, for some different levels of occlusion, each 3D bar chart displays, along the z-axis, the percentage of common points between the eight persistence diagrams associated with each of the 70 occluded shapes ( $\mathrm{x}$ axis), and the eight persistence diagrams associated with each of the 70 original ones (y-axis). We see that, for each occluded shape, the highest bar is always on the diagonal, that is, where the occluded object is compared with the corresponding original one.
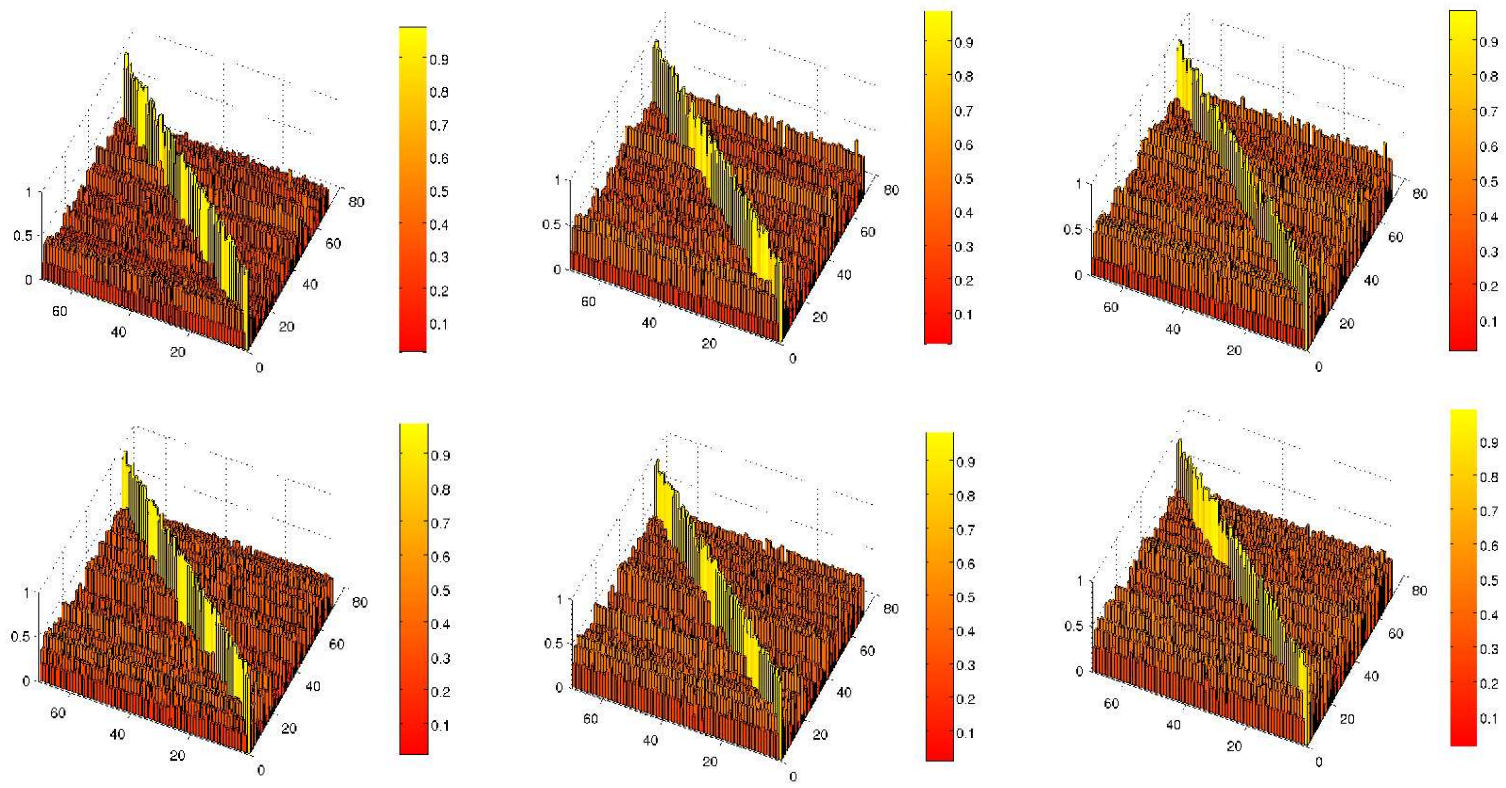

Table 1: 3D bar charts displaying the percentage of common points (z-axis) between the persistence diagrams of the 70 occluded shapes (x-axis) and the persistence diagrams of the 70 original ones (y-axis) correspondingly ordered. First row: Shapes are occluded from the top by $20 \%$ (column 1), by $40 \%$ (column 2), by $60 \%$ (column 3). Second row: Shapes are occluded from the left by $20 \%$ (column 1 ), by $40 \%$ (column 2), by $60 \%$ (column 3 ).

Moreover, to display the robustness of points of a persistence diagram under occlusion, three particular instances of our dataset images are shown in Tables 2-4 (first column) with their persistence diagrams with respect to the second group of four filtrating functions (the next-to-last column). The chosen images are characterized by different homological features, which will be changed in the presence of occlusion. For example, the "camel" in Table 2 is a connected object without holes, but it may happen that the occlusion makes the first homological group non-trivial (see second row, first column). On the other hand, Table 3 shows a "frog", which is a connected object with several holes. The different percentages of occluded area can create some new holes or destroy them (see rows 3-4). Eventually, the "pocket watch", represented in Table 4, is primarily characterized by several connected components, whose number decreases as the occluding area increases. This results in a reduction of the number of points at infinity in its persistence diagrams. In spite of these topological changes, it can easily 

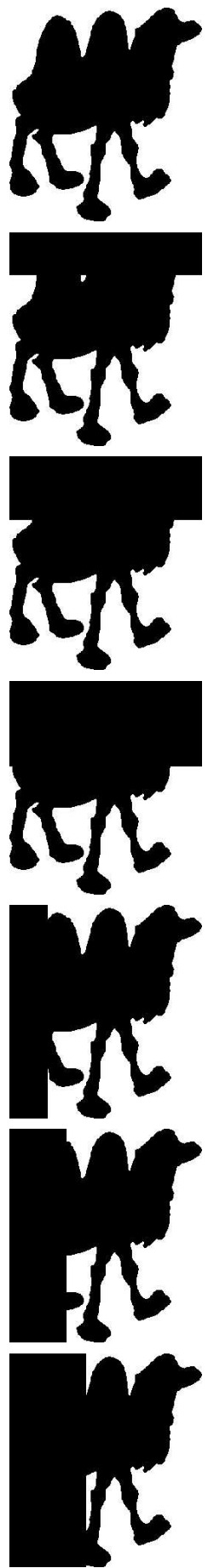
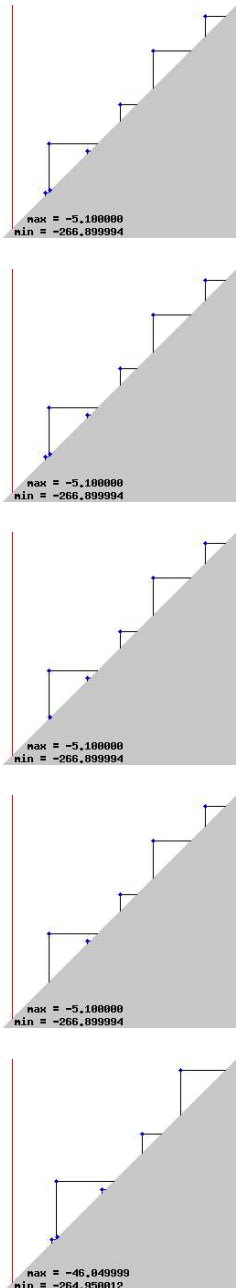

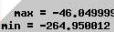
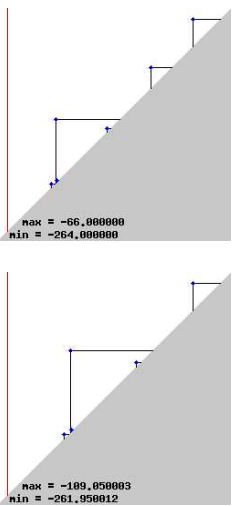

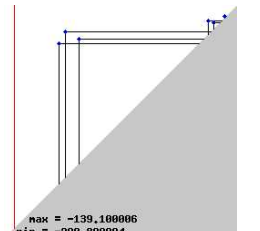

$\min =-290.999994$
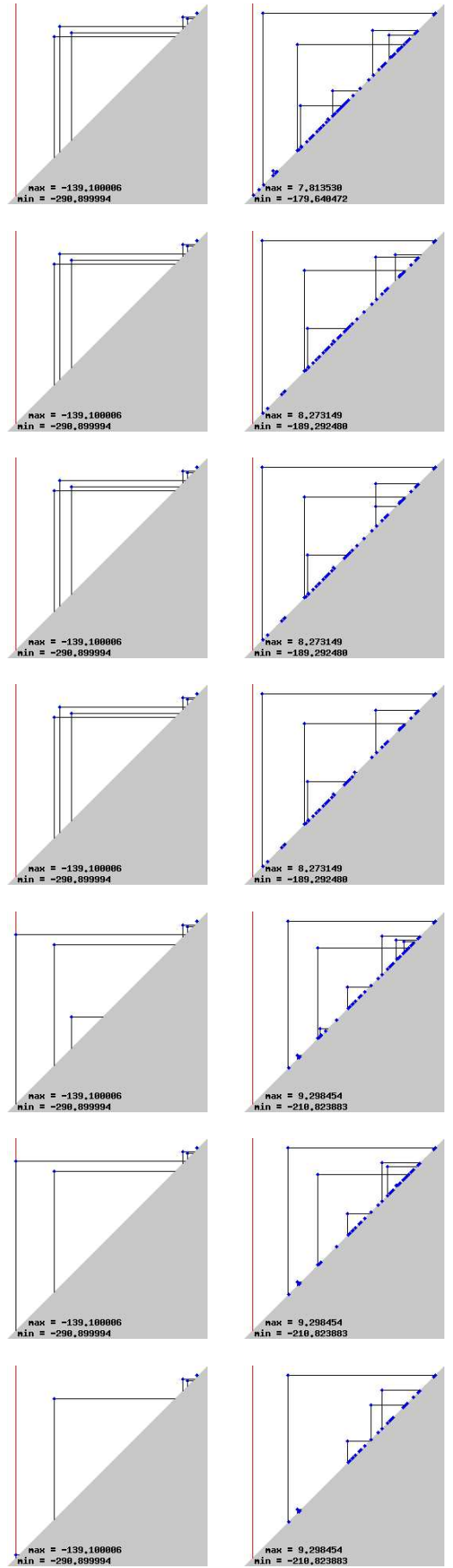

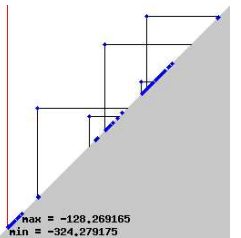

$\operatorname{Rin}=-324,227975$
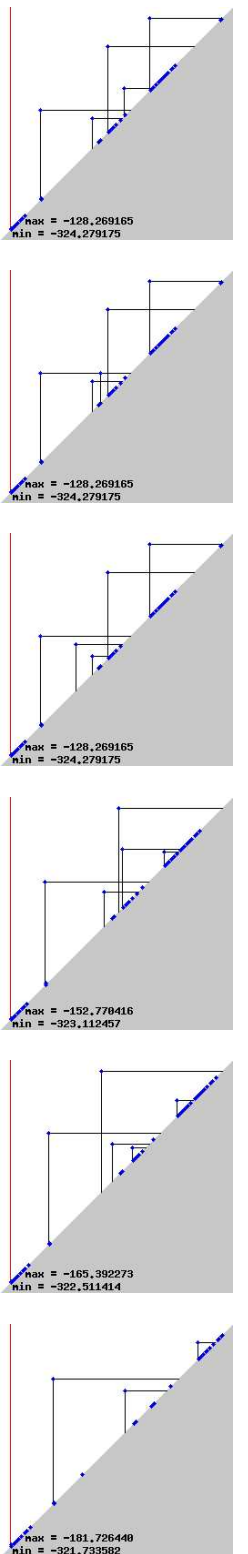

Table 2: The first column: (row 1) original "camel" shape, (rows 2-4) occluded from the top by $20 \%, 30 \%, 40 \%$, (row 5-7) occluded from the left by $20 \%, 30 \%, 40 \%$. From second column onwards: the points of persistence diagrams related to filtrating functions defined as minus distances from four lines rotated by $0, \pi / 4, \pi / 2,3 \pi / 4$, with respect to the horizontal position. 

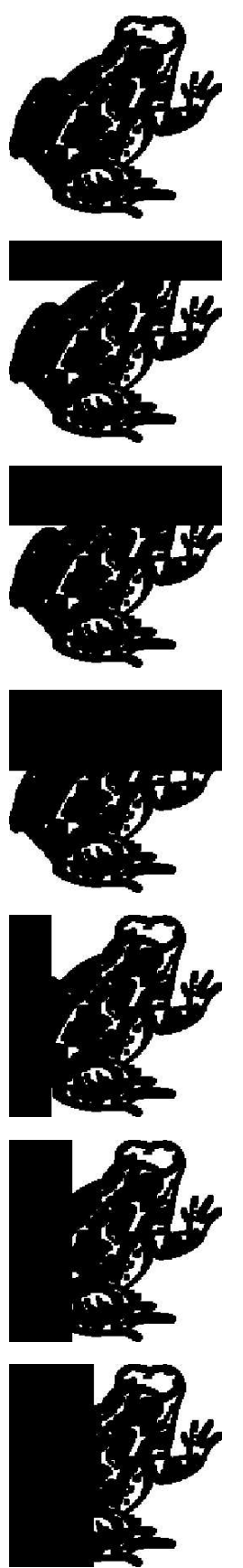
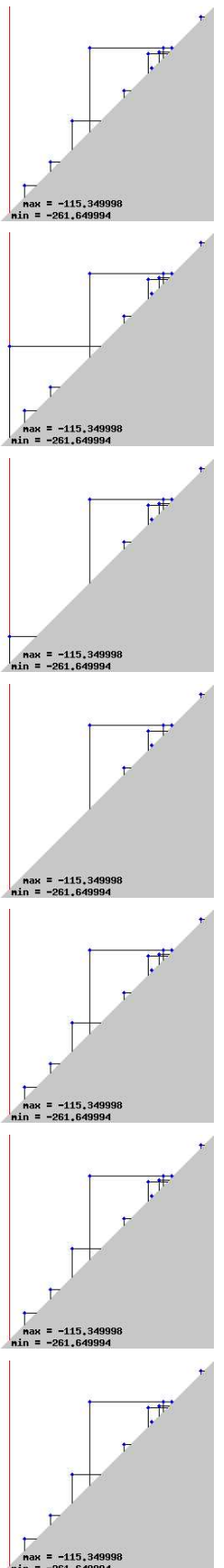

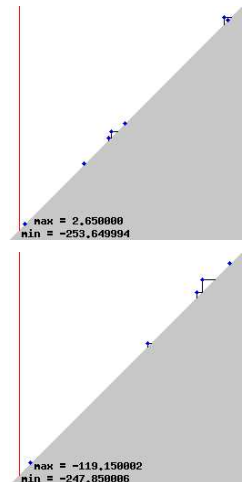

$\max =-119.150002$
$\min =-247.858806$

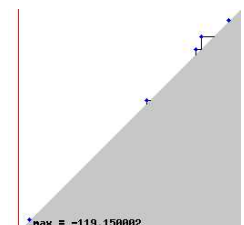

$\max =-119.150003$
$\min =-247.856806$
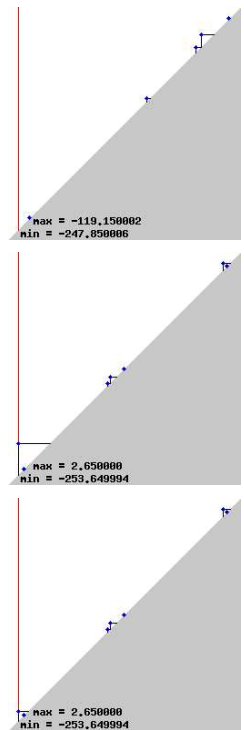

nin $=-253.649994$

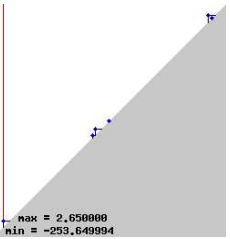

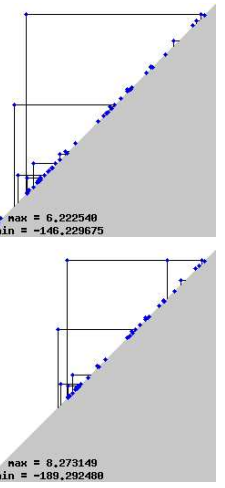
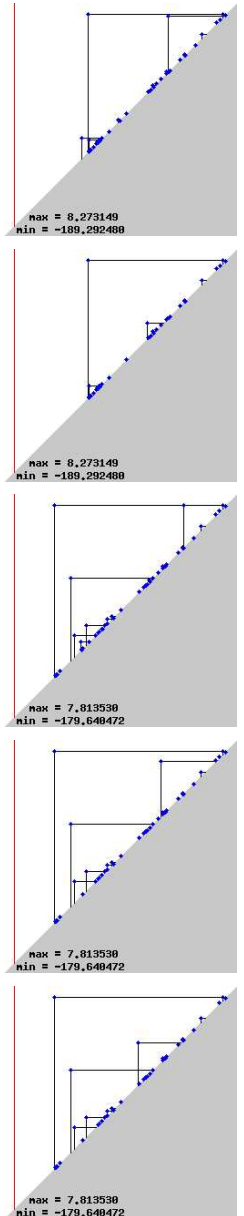
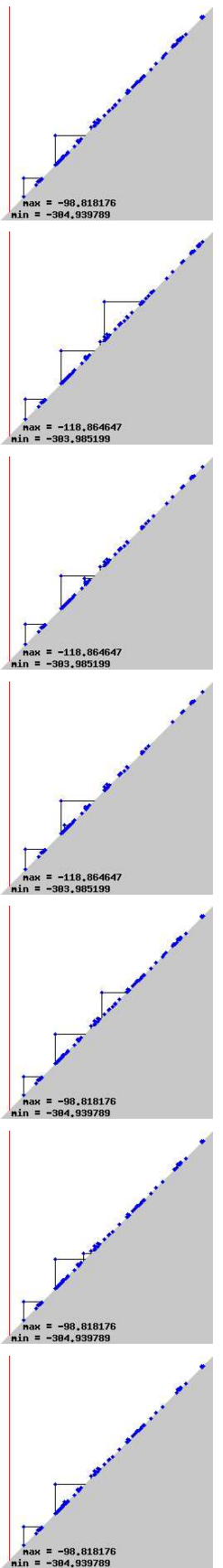

Table 3: The first column: (row 1) original "frog" shape, (rows 2-4) occluded from the top by $20 \%, 30 \%, 40 \%$, (row 5-7) occluded from the left by $20 \%, 30 \%, 40 \%$. From second column onwards: the points of persistence diagrams related to filtrating functions defined as minus distances from four lines rotated by $0, \pi / 4, \pi / 2,3 \pi / 4$, with respect to the horizontal position. 

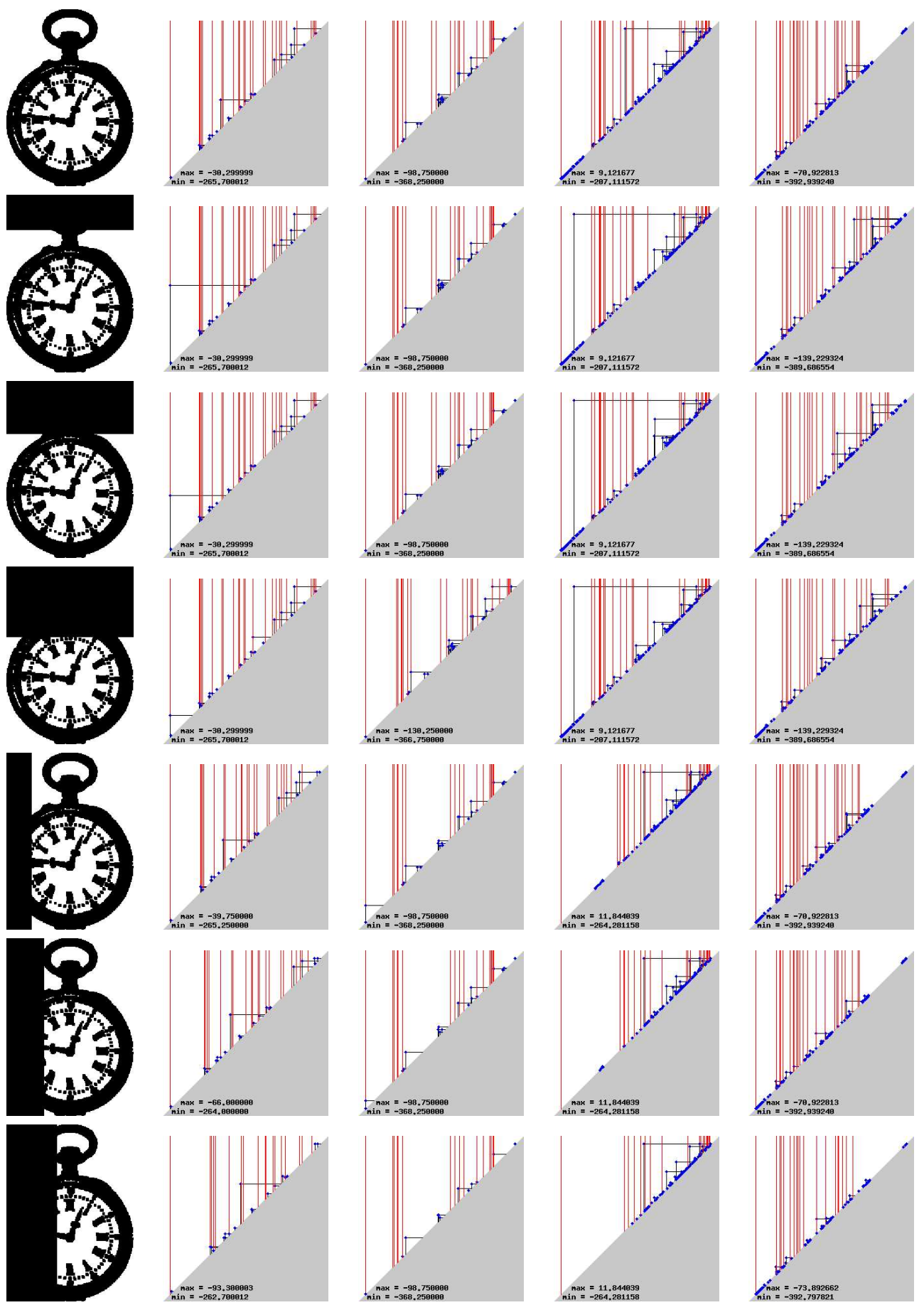

Table 4: The first column: (row 1) original "pocket watch" shape, (rows 2-4) occluded from the top by 20\%, 30\%, 40\%, (row 5-7) occluded from the left by $20 \%, 30 \%, 40 \%$. From second column onwards: the points of persistence diagrams related to filtrating functions defined as minus distances from four lines rotated by $0, \pi / 4, \pi / 2,3 \pi / 4$, with respect to the horizontal position. 
be seen that, given a filtrating function, even if the persistence diagram related to a shape and the persistence diagram related to the occluded shape are defined by different sets of points, because of occlusion, a common subset of these is present, making possible a partial match between them.

The second experiment is a recognition test for occluded shapes by comparison of points of the associated persistence diagrams. In this case the rectangular-shaped occlusion is not visible (see Table 5). When the original shape is disconnected by the occlusion, we retain only the connected component of greatest area. With reference to the notation used in our theoretical setting, here we are considering $X$ as the original shape, $A$ as the the occluded shape, and $B$ as the invisible part of $X$.

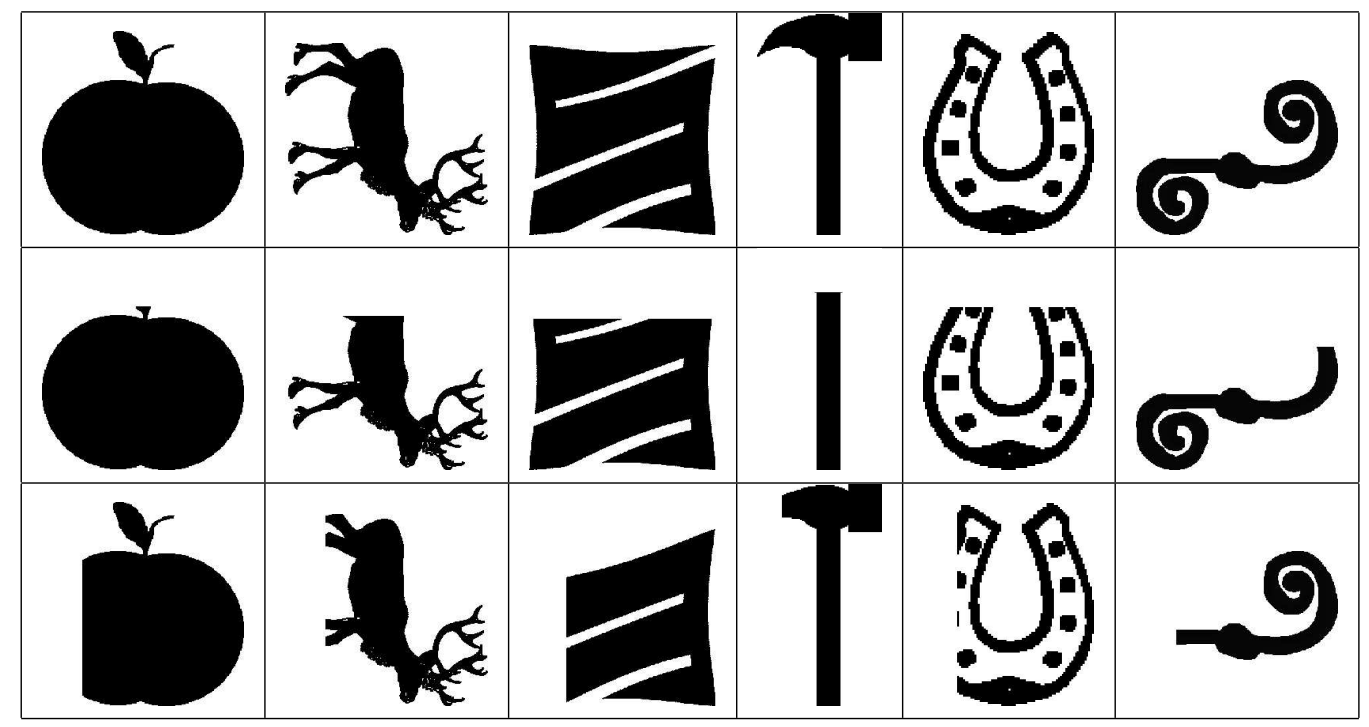

Table 5: The first row: some instances from the MPEG-7 dataset; the second and third rows: by $20 \%$ occluded from the top and from the left, respectively.

Rate of recognition with horizontal occlusions

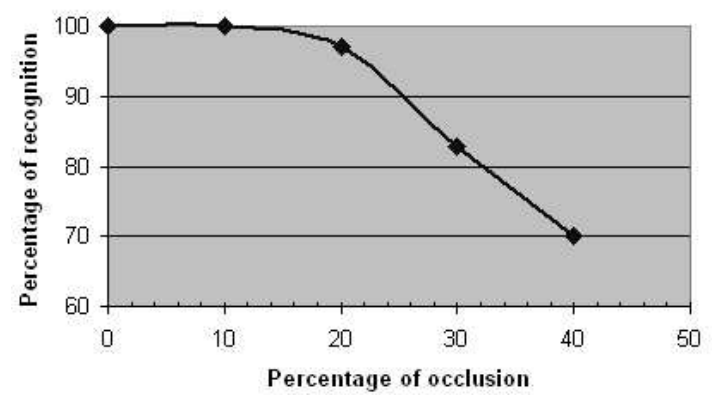

Rate of recognition with vertical occlusions

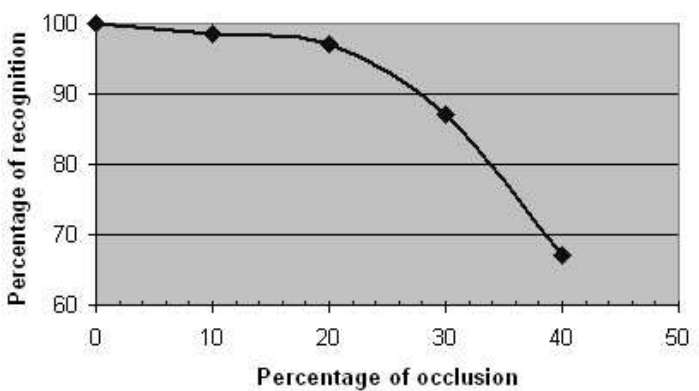

Table 6: The leftmost (rightmost, respectively) graph describes the recognition trend when the occluded area from the top (left, respectively) increases.

By varying the amount of occluded area, we compare each occluded shape with each of the 70 original shapes. 
Comparison is performed by calculating the sum of the eight Hausdorff distances between the sets of points of the persistence diagrams associated with the corresponding eight filtrating functions. Then each occluded shape is assigned to the class of its nearest neighbor among the original shapes.

In Table 6, two graphs describe the rate of correct recognition in the presence of an increasing percentage of occlusion. The leftmost graph is related to the occlusion from the top, while the rightmost one is related to the same occlusion from the left.

\section{Discussion}

The main contribution of this paper is the development of a Mayer-Vietoris formula for persistent homology. In plain words, according to this formula, persistent homology groups of a space $X$ are determined by the persistent homology groups of two subspaces $A$ and $B$ such that $A \cup B=X$, the persistent homology groups of their intersection, and the kernels of certain homomorphisms. A research area that could stem from this result, as suggested by one of the referees, concerns the development of a divide-and-conquer algorithm for persistent homology computation. However a difficulty in this direction is that the kernels involved in our formula seem to be not algorithmically computable.

A further result of this paper is that the Mayer-Vietoris formula for persistent homology groups of $X, A$, and $B$ is inherited by the multiplicities of points of the associated persistence diagrams. Therefore, using persistence diagrams, recognition of a shape from partial information becomes an easy task. Indeed, recognition is achieved simply by matching those persistence diagrams with the largest common subset of points (as in the experiment in Table 1).

In practice, however, shapes may undergo other deformations due to perspective, articulations, or noise, for instance. As a consequence of these alterations, points of persistence diagrams may move. Anyway, small continuous changes in shape induce small displacements in persistence diagrams $[5,6,7]$.

It has to be expected that, when a shape is not only occluded but also deformed, it will not be possible to find a common subset between the persistence diagram of the original shape and that of the occluded one, since the deformation has slightly changed the points position. At the same time, however, the Hausdorff distance between the persistence diagram of the original shape and the persistence diagram of the occluded shape will not need to be small, because it takes into account the total number of points, including, for example, those inherited from the occluding pattern (as in the experiment in Table 6).

The present work is a necessary step, in view of the more general goal of recognizing shapes in the presence of both partial information and deformations. The development of a method to measure the partial matching of persistence diagrams that do not exactly overlap but are slightly shifted would be desirable.

\section{Acknowledgments}

In the original version of this paper the Mayer-Vietoris formula was obtained only for the case $k=0$. The stronger result presented here, that holds for arbitrary values of $k$, was obtained in a later revision of the paper. We are grateful to one of the referees for stimulating and encouraging us to search in this direction.

As pointed out to us by C. L. Schochet, there is another homology theory defined for all compact spaces and for any coefficient group $G$ that always gives an exact Mayer-Vietoris sequence, called Steenrod homology. Under our assumptions on $G$, Steenrod homology coincides with Čech homology. We are grateful to C. L. Schochet for this interesting observation.

We wish to thank F. Cagliari, M. Grandis, for their helpful suggestions and P. Frosini for suggesting the example in Figure $5(a)$. Thanks also to A. Cerri and F. Medri for their invaluable help with the software. However, the authors are solely responsible for any possible errors.

Finally, we wish to express our gratitude to M. Ferri and P. Frosini for their indispensable support and friendship. 


\section{A Appendix: Čech homology and Mayer-Vietoris sequence}

The Čech approach is based on a way to generate simplicial complexes from finite covering of a space, by taking the nerve of the covering. Since the nerve of a covering is a simplicial complex, we can compute its homology groups by the usual techniques. Refining the covering one obtains an inverse system of finite triangulations that approximate a space. A Čech homology group is the inverse limit of such a system. Detailed descriptions of Cech homology theory can be found in [10]. Here we briefly survey the subject, focusing on the Mayer-Vietoris sequence of Čech homology.

Given a compact Hausdorff space $X$, let $\Sigma(X)$ denote the family of all finite coverings of $X$ by open sets. The coverings in $\Sigma(X)$ will be denoted by script letters $\mathscr{U}, \mathscr{V}, \ldots$ and the open sets in a covering by italic capitals $U, V, \ldots$ An element $\mathscr{U}$ of $\Sigma(X)$ may be considered as a simplicial complex if we define vertex to mean open set $U$ in $\mathscr{U}$ and agree that a subcollection $U_{0}, \ldots, U_{k}$ of such vertices constitutes a $k$-simplex if and only if the intersection $\bigcap_{i=0}^{k} U_{i}$ is not empty. The resulting complex is known as the nerve of the covering $\mathscr{U}$.

Given a covering $\mathscr{U}$ in $\Sigma(X)$, we may define the chain groups $C_{k}(\mathscr{U}, G)$, the cycle groups $Z_{k}(\mathscr{U}, G)$, the boundary groups $B_{k}(\mathscr{U}, G)$, and the homology groups $H_{k}(\mathscr{U}, G)$.

The collection $\Sigma(X)$ of finite open coverings of a space $X$ may be partially ordered by refinement. A covering $\mathscr{V}$ refines the covering $\mathscr{U}$, and we write $\mathscr{U}<\mathscr{V}$, if every element of $\mathscr{V}$ is contained in some element of $\mathscr{U}$. It turns out that $\Sigma(X)$ is a direct set under refinement.

If $\mathscr{U}<\mathscr{V}$ in $\Sigma(X)$, then there is a simplicial mapping $\pi_{\mathscr{U} \mathscr{V}}$ of $\mathscr{V}$ into $\mathscr{U}$ called a projection. This is defined by taking $\pi_{\mathscr{U} \mathscr{V}}(V), V \in \mathscr{V}$, to be any (fixed) element $U$ of $\mathscr{U}$ such that $V$ is contained in $U$. There may be many projections of $\mathscr{V}$ into $\mathscr{U}$. Each projection $\pi_{\mathscr{U} \mathscr{V}}$ induces a chain mapping of $C_{k}(\mathscr{V}, G)$ into $C_{k}(\mathscr{U}, G)$, still denoted by $\pi_{\mathscr{U} \mathscr{V}}$, and this in turn induces homomorphisms ${ }_{*} \pi_{\mathscr{U} \mathscr{V}}$ of $H_{k}(\mathscr{V}, G)$ into $H_{k}(\mathscr{U}, G)$. If $\mathscr{U}<\mathscr{V}$ in $\Sigma(X)$, then it can be proved that any two projections of $\mathscr{V}$ into $\mathscr{U}$ induce the same homomorphism of $H_{k}(\mathscr{V}, G)$ into $H_{k}(\mathscr{U}, G)$.

Taking the limit of the inverse system $\left(H_{k}(\mathscr{V}, G){ }_{{ }_{*}} \pi_{\mathscr{U} \mathscr{V}}\right)$ one obtains the $k$ th Čech homology group.

In general, Čech homology theory has all the axioms of homology theories except the exactness axiom. However, if some assumptions are made on the considered spaces and coefficients, this axiom also holds. Indeed, in [10, Chap. IX, Thm. 7.6] (see also [16]), we read the following result concerning the sequence of a pair $(X, A)$

$$
\cdots \longrightarrow \check{H}_{k+1}(X, A) \stackrel{\partial_{k}}{\longrightarrow} \check{H}_{k}(A) \stackrel{i_{k}}{\longrightarrow} \check{H}_{k}(X) \stackrel{j_{k}}{\longrightarrow} \check{H}_{k}(X, A) \stackrel{\partial_{k-1}}{\longrightarrow} \cdots \longrightarrow \check{H}_{0}(X, A) \longrightarrow 0
$$

which, in general, is only of order 2 (this means that the composition of any two successive homomorphisms of the sequence is zero, i.e. im $\subseteq$ ker).

Theorem A.1. [10, Chap. IX, Thm. 7.6] If $(X, A)$ is compact and $G$ is a vector space over a field, then the homology sequence of the pair $(X, A)$ is exact.

It follows that, if $(X, A)$ is compact and $G$ is a vector space over a field, Čech homology satisfies all the axioms of homology theories, and therefore all the general theorems in [10, Chap. I] also hold for Čech homology.

It is important to observe that Čech homology is characterized by a further axiom besides the ordinary ones, that is the continuity axiom. This axiom states that Čech homology commutes with inverse limits [10]. This makes Čech homology different from singular homology since, for example, the first sees the Warsaw circle as a non-trivial space, while the latter does not.

As a consequence of the continuity property, Čech homology satisfies a stronger form of the excision axiom. This, in particular, implies that, in Čech theory, every compact triad is a proper triad [10, Chap. IX].

Hence, using [10, Chap. I, Thm. 15.3], we deduce the following result:

Theorem A.2. Let $(X, A, B)$ be a compact triad and $G$ be a vector space over a field. The Mayer-Vietoris sequence of $(X, A, B)$ with $X=A \cup B$

$$
\cdots \longrightarrow \check{H}_{k+1}(X) \stackrel{\delta_{k}}{\longrightarrow} \check{H}_{k}(A \cap B) \stackrel{\alpha_{k}}{\longrightarrow} \check{H}_{k}(A) \oplus \check{H}_{k}(B) \stackrel{\beta_{k}}{\longrightarrow} \check{H}_{k}(X) \stackrel{\delta_{k-1}}{\longrightarrow} \cdots \longrightarrow \check{H}_{0}(X) \longrightarrow 0
$$


is exact.

Concerning homomorphisms between Mayer-Vietoris sequences, from [10, Chap. I, Thm. 15.4], the following result holds.

Theorem A.3. If $(X, A, B)$ and $(Y, C, D)$ are compact triads, $X=A \cup B, Y=C \cup D$, and $f:(X, A, B) \rightarrow(Y, C, D)$ is a map of one compact triad into another, then $f$ induces a homomorphism of the Mayer-Vietoris sequence of $(X, A, B)$ into that of $(Y, C, D)$ such that commutativity holds in the diagram

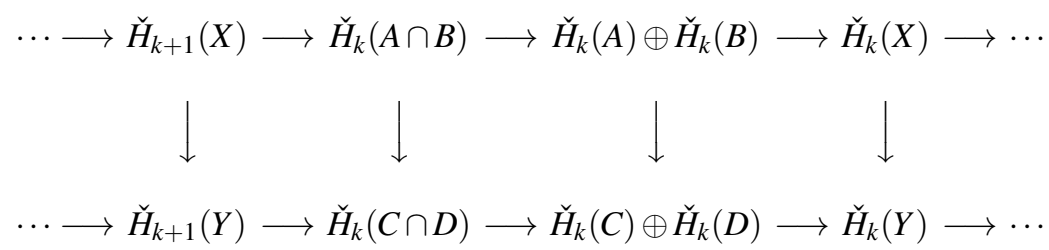

A relative form of the Mayer-Vietoris sequence, different from the one proposed in [10], is useful in the present paper. In order to obtain this sequence, we can adapt the construction explained in [14] to Čech homology and obtain the following result.

Theorem A.4. If $(X, A, B)$ and $(Y, C, D)$ are compact triads with $X=A \cup B, Y=C \cup D, Y \subseteq X, C \subseteq A, D \subseteq B$, then there is a relative Mayer-Vietoris sequence of homology groups with coefficients in a vector space $G$ over a field

$$
\cdots \longrightarrow \check{H}_{k+1}(X, Y) \longrightarrow \check{H}_{k}(A \cap B, C \cap D) \longrightarrow \check{H}_{k}(A, C) \oplus \check{H}_{k}(B, D) \longrightarrow \check{H}_{k}(X, Y) \longrightarrow \cdots
$$

that is exact.

Proof. Given a covering $\mathscr{U}$ of $\Sigma(X)$, we may consider the relative simplicial homology groups $H_{k}\left(\mathscr{U}, \mathscr{U}_{Y}\right)$, $H_{k}\left(\mathscr{U}_{A}, \mathscr{U}_{C}\right), H_{k}\left(\mathscr{U}_{B}, \mathscr{U}_{D}\right), H_{k}\left(\mathscr{U}_{A \cap B}, \mathscr{U}_{C \cap D}\right)$, for every $k \in \mathbb{Z}$. For these groups the relative Mayer-Vietoris sequence

$$
\cdots \longrightarrow H_{k+1}\left(\mathscr{U}, \mathscr{U}_{Y}\right) \longrightarrow H_{k}\left(\mathscr{U}_{A \cap B}, \mathscr{U}_{C \cap D}\right) \longrightarrow H_{k}\left(\mathscr{U}_{A}, \mathscr{U}_{C}\right) \oplus H_{k}\left(\mathscr{U}_{B}, \mathscr{U}_{D}\right) \longrightarrow H_{k}\left(\mathscr{U}, \mathscr{U}_{Y}\right) \longrightarrow \cdots
$$

is exact (cf. [14, page 152]).

We now recall that the $k$ th Čech homology group of a pair of spaces $(X, Y)$ over $G$ is the inverse limit of the system of groups $\left\{H_{k}\left(\mathscr{U}, \mathscr{U}_{Y}, G\right)_{, *} \pi_{\mathscr{U} \mathscr{Y}}\right\}$ defined on the direct set of all open coverings of the pair $(X, Y)$ (cf. [10, Chap. IX, Thm. 3.2 and Def. 3.3]). The claim is proved recalling that, given an inverse system of exact lower sequences, where all the terms of the sequence belong to the category of vector spaces over a field, the limit sequence is also exact (cf. [10, Chap. VIII, Thm. 5.7] and [16]).

The following result, concerning homomorphisms of relative Mayer-Vietoris exact sequences, holds. We omit the proof, which can be obtained in a standard way.

Theorem A.5. If $(X, A, B),(Y, C, D),\left(X^{\prime}, A^{\prime}, B^{\prime}\right),\left(Y^{\prime}, C^{\prime}, D^{\prime}\right)$ are compact triads with $X=A \cup B, Y=C \cup D$, $Y \subseteq X, C \subseteq A, D \subseteq B$, and $X^{\prime}=A^{\prime} \cup B^{\prime}, Y^{\prime}=C^{\prime} \cup D^{\prime}, Y^{\prime} \subseteq X^{\prime}, C^{\prime} \subseteq A^{\prime}, D^{\prime} \subseteq B^{\prime}$, and $f: X \rightarrow X^{\prime}$ is a map such that $f(Y) \subseteq Y^{\prime}, f(A) \subseteq A^{\prime}, f(B) \subseteq B^{\prime}, f(C) \subseteq C^{\prime}, f(D) \subseteq D^{\prime}$, then $f$ induces a homomorphism of the relative Mayer-Vietoris sequences such that commutativity holds in the diagram

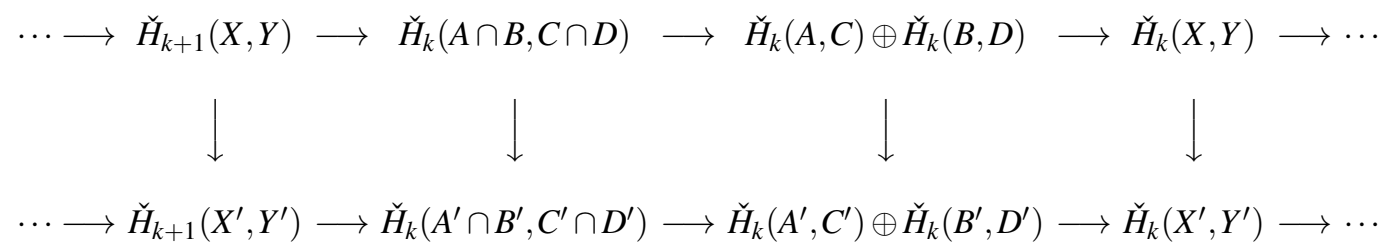




\section{B Appendix: Relating homological critical values and points of persis- tence diagrams}

In this section we show the link between homological critical values and points of persistence diagrams. To the best of our knowledge, this connection, which is rather intuitive, has not been proved elsewhere. Moreover, although in the case of tame functions (i.e., functions with at most a finite number of homological critical values, and whose lower level sets have finitely generated homology groups) it may be trivial, it is unfortunately not so in the case considered here.

Homological critical values have been introduced in [5], and intuitively correspond to values where the lower level sets of the function undergo a topological change. We prove that the coordinates of points of persistence diagrams are always homological critical values, while the converse is true only assuming that the number of homological critical values is finite (Proposition B.4). Indeed, in general, there may exist homological critical values not generating points with a positive multiplicity (Remark B.5).

Definition B.1. Let $\varphi: X \rightarrow \mathbb{R}$ be a continuous function. $A$ homological $k$-critical value of $\varphi$ is a real number $w$ such that, for every sufficiently small $\varepsilon>0$, the map $\imath_{k}^{w-\varepsilon, w+\varepsilon}: \breve{H}_{k}\left(X_{w-\varepsilon}\right) \rightarrow \breve{H}_{k}\left(X_{w+\varepsilon}\right)$ induced by inclusion is not an isomorphism.

Lemma B.2. [3, Lemma 2.2] The rank of persistent homology groups $\operatorname{rk} \breve{H}_{k}^{u, v}(X, \varphi)$ as an integer-valued function of the proper points $(u, v) \in \Delta^{*}$ is non-decreasing in the variable $u$ and non-increasing in the variable $v$.

Lemma B.3. The following statements hold:

(a) $\bar{u} \in \mathbb{R}$ is the abscissa of a point (either proper or at infinity) of $D_{k}(X, \varphi)$ if and only if there exists $v>\bar{u}$ such that $\mathrm{rk} \breve{H}_{k}^{\bar{u}-\varepsilon, v}(X, \varphi)<\operatorname{rk} \breve{H}_{k}^{\bar{u}+\varepsilon, v}(X, \varphi)$ for every $\varepsilon>0$ sufficiently small;

(b) $\bar{v} \in \mathbb{R}$ is the ordinate of a proper point of $D_{k}(X, \varphi)$ if and only if there exists $u<\bar{v}$ such that $\mathrm{rk} \check{H}_{k}^{u, \bar{v}-\varepsilon}(X, \varphi)>$ $\operatorname{rk} \breve{H}_{k}^{u, \bar{v}+\varepsilon}(X, \varphi)$ for every $\varepsilon>0$ sufficiently small.

Proof. Let us prove the direct statement of $(a)$ (the proof of the direct statement of $(b)$ is analogous). Let $p=$ $(\bar{u}, \bar{v})$ be a proper point, with $\mu_{(X, \varphi), k}(p)>0$. Definition 2.2 implies that $\operatorname{rk} \breve{H}_{k}^{\bar{u}+\varepsilon, \bar{v}-\varepsilon}(X, \varphi)-\operatorname{rk} \breve{H}_{k}^{\bar{u}-\varepsilon, \bar{v}-\varepsilon}(X, \varphi)>$ $\operatorname{rk} \breve{H}_{k}^{\bar{u}+\varepsilon, \bar{v}+\varepsilon}(X, \varphi)-\operatorname{rk} \breve{H}_{k}^{\bar{u}-\varepsilon, \bar{v}+\varepsilon}(X, \varphi)$ for every $\varepsilon>0$ sufficiently small. Since by Lemma B.2, $\operatorname{rk} \breve{H}_{k}^{\bar{u}+\varepsilon, \bar{v}+\varepsilon}(X, \varphi)-$ $\operatorname{rk} \breve{H}_{k}^{\bar{u}-\varepsilon, \bar{v}+\varepsilon}(X, \varphi) \geq 0$ for every $\varepsilon>0$ sufficiently small, the claim immediately follows. If $\bar{u}$ is the abscissa of a point at infinity of $D_{k}(X, \varphi)$, the same reasoning can be applied, but this time using Definition 2.3.

The converse statement of $(a)((b)$, respectively) can be proved by extending [12, Lemma $3(i)]$ ([12, Lemma 3 (ii)], respectively) to all homology degrees.

Proposition B.4. The coordinates of points of $D_{k}(X, \varphi)$ are homological $k$-critical values of $\varphi$. Moreover, if $\varphi$ admits at most a finite number of homological $k$-critical values, then the converse statement holds, too.

Proof. Let us prove that abscissas of points of $D_{k}(X, \varphi)$ are homological $k$-critical values of $\varphi$.

If $\bar{u} \in \mathbb{R}$ is the abscissa of a point (either proper or at infinity) of $D_{k}(X, \varphi)$, then, by the previous Lemma B.3(a), there exists $v>\bar{u}$ such that $\operatorname{rkim} \imath_{k}^{\bar{u}+\varepsilon, v}=\operatorname{rk} \breve{H}_{k}^{\bar{u}+\varepsilon, v}(X, \varphi)>\operatorname{rk} \breve{H}_{k}^{\bar{u}-\varepsilon, v}(X, \varphi)=\operatorname{rkim} \imath_{k}^{\bar{u}-\varepsilon, v}$ for every sufficiently small $\varepsilon>0$. Observing that $\imath_{k}^{\bar{u}-\varepsilon, v}=\imath_{k}^{\bar{u}+\varepsilon, v} \circ l_{k}^{\bar{u}-\varepsilon, \bar{u}+\varepsilon}$, we deduce that $\imath_{k}^{\bar{u}-\varepsilon, \bar{u}+\varepsilon}$ is not an isomorphism for any $\varepsilon>0$ sufficiently small.

The proof that ordinates of points of $D_{k}(X, \varphi)$ are homological $k$-critical values of $\varphi$ can be seen similarly, by using Lemma B.3(b).

To prove the second assertion, let $w$ be a homological $k$-critical value of $\varphi$. Then, by Definition B.1, for every $\varepsilon>0$ sufficiently small, $\imath_{k}^{w-\varepsilon, w+\varepsilon}$ is not an isomorphism. Let us show that the following statements hold:

(i) If $\imath_{k}^{w-\varepsilon, w+\varepsilon}$ is not surjective for any sufficiently small $\varepsilon>0$, then $w$ is the abscissa of a point $p$ (either proper or at infinity), with $\mu_{(X, \varphi), k}(p)>0$; 
(ii) If $\imath_{k}^{w-\varepsilon, w+\varepsilon}$ is surjective for every sufficiently small $\varepsilon>0$, then $w$ is the ordinate of a proper point $p$, with $\mu_{(X, \varphi), k}(p)>0$.

Let us prove $(i)$. Since we are assuming the presence of at most a finite number of homological $k$-critical values of $\varphi$, there surely exists $v>w$ such that, for every sufficiently small $\varepsilon>0, v>w+\varepsilon$ and $\imath_{k}^{w+\varepsilon, v}: \breve{H}_{k}\left(X_{w+\varepsilon}\right) \rightarrow$ $\breve{H}_{k}\left(X_{v}\right)$ is an isomorphism. Hence, we deduce that $\operatorname{rkim} \imath_{k}^{w-\varepsilon, v}=\operatorname{rkim}\left(\imath_{k}^{w+\varepsilon, v} \circ \imath_{k}^{w-\varepsilon, w+\varepsilon}\right)=\operatorname{rkim} \imath_{k}^{w-\varepsilon, w+\varepsilon}<$ $\operatorname{rk} \breve{H}_{k}\left(X_{w+\varepsilon}\right)=\operatorname{rkim} \imath_{k}^{w+\varepsilon, v}$, where the second equality holds because $\imath_{k}^{w+\varepsilon, v}$ is an isomorphism, the inequality holds because $\operatorname{rkim} \imath_{k}^{w-\varepsilon, w+\varepsilon}<+\infty$ and we are assuming $\imath_{k}^{w-\varepsilon, w+\varepsilon}$ not surjective, and the last equality holds again because $\imath_{k}^{w+\varepsilon, v}$ is an isomorphism. Thus, $\operatorname{rk} \breve{H}_{k}^{w-\varepsilon, v}(X)<\operatorname{rk} \breve{H}_{k}^{w+\varepsilon, v}(X)$ for every $\varepsilon>0$ sufficiently small, and, by Lemma B.3 $(a)$, the claim follows.

As for $(i i)$, the assumption that $\varphi$ has at most a finite number of homological $k$-critical values implies the existence of $u<w$ such that, for every sufficiently small $\varepsilon>0, u<w-\varepsilon$ and $\imath_{k}^{u, w-\varepsilon}: \check{H}_{k}\left(X_{u}\right) \rightarrow \breve{H}_{k}\left(X_{w-\varepsilon}\right)$ is an isomorphism. Moreover, since $\imath_{k}^{w-\varepsilon, w+\varepsilon}$ is surjective, and $w$ is a homological $k$-critical value, it necessarily follows that $\imath_{k}^{w-\varepsilon, w+\varepsilon}$ is not injective. Therefore, we have that $\operatorname{rkim} \imath_{k}^{u, w+\varepsilon}=\operatorname{rkim}\left(\imath_{k}^{w-\varepsilon, w+\varepsilon} \circ \imath_{k}^{u, w-\varepsilon}\right)=$ $\operatorname{rkim} \imath_{k}^{w-\varepsilon, w+\varepsilon}<\operatorname{rk} \check{H}_{k}\left(X_{w-\varepsilon}\right)=\operatorname{rkim} \imath_{k}^{u, w-\varepsilon}$, where the second equality holds because $\imath_{k}^{u, w-\varepsilon}$ is an isomorphism, the inequality holds because $\operatorname{rkim} \imath_{k}^{w-\varepsilon, w+\varepsilon}<+\infty$ and we are assuming $\imath_{k}^{w-\varepsilon, w+\varepsilon}$ not injective, and the last equality holds again because $\imath_{k}^{u, w-\varepsilon}$ is an isomorphism. Thus, it follows that $\operatorname{rk} \breve{H}_{k}^{u, w-\varepsilon}(X)>\operatorname{rk} \breve{H}_{k}^{u, w+\varepsilon}(X)$ for every $\varepsilon>0$ sufficiently small, implying the claim by Lemma B.3(b).

Dropping the assumption that the number of homological $k$-critical values of $\varphi$ is finite, the converse of the first statement of Proposition B.4 is false, as the following remark states.

Remark B.5. From the condition that $w$ is a homological $k$-critical value of a continuous function $\varphi: X \rightarrow \mathbb{R}$, it does not follow that $w$ is a coordinate of one of the points belonging to $D_{k}(X, \varphi)$.
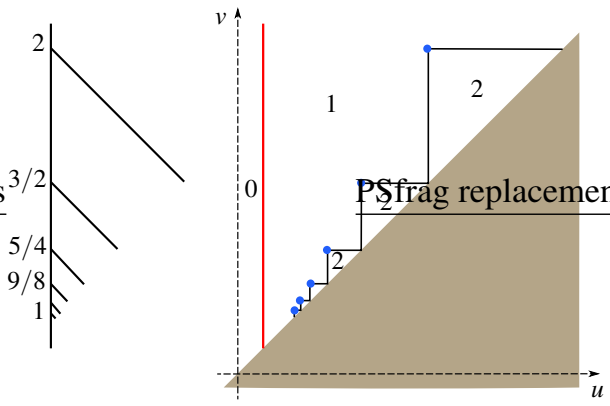

(a)

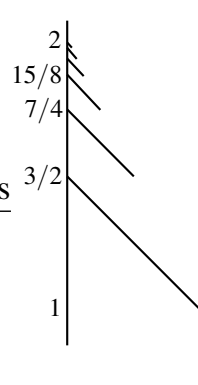

(b)

Figure 5: Two examples showing the existence of a real number $w$ that is a homological 0-critical value of $\varphi: X \rightarrow \mathbb{R}$ but not the coordinate of any point of $D_{0}(X, \varphi)$.

Two different examples, shown in Figure 5, support our claim.

Let us describe the first example (see Figure 5(a)). Let $X$ be the topological space obtained by adding an infinite number of branches to a vertical segment, each one sprouting at the height where the previous expires. These heights are chosen according to the sequence $\left(1+\frac{1}{2^{n}}\right)_{n \in \mathbb{N}}$, converging to 1 . The filtering function $\varphi$ is the height function. The persistence diagram $D_{0}(X, \varphi)$ is displayed on the right side of $X$. In this case, $w=1$ is a homological 0-critical value. Indeed, for $w=1$, it holds that $\operatorname{rk} \breve{H}_{0}\left(X_{w-\varepsilon}\right)=1$ while $\operatorname{rk} \breve{H}_{0}\left(X_{w+\varepsilon}\right)=2$ for every sufficiently small $\varepsilon>0$. On the other hand, let us show that $w$ is not one of the coordinates of any point in $D_{0}(X, \varphi)$. For every $v>w$, and for every small enough $\varepsilon>0$, it holds that $\operatorname{rk} \breve{H}_{0}^{w+\varepsilon, v}(X)=\operatorname{rk} \breve{H}_{0}^{w-\varepsilon, v}(X)=1$. 
Therefore, by Lemma B.3(a), it follows that $w$ is not the abscissa of any point belonging to $D_{0}(X, \varphi)$. Moreover, that $w$ is not the ordinate of any proper point of $D_{0}(X, \varphi)$ is a trivial consequence of Lemma B.3(b) since, for every $u<w$, and every small enough $\varepsilon>0$, it holds that $\operatorname{rk} \breve{H}_{0}^{u, w-\varepsilon}(X)=\operatorname{rk} \breve{H}_{0}^{u, w+\varepsilon}(X)=0$ or $\operatorname{rk} \breve{H}_{0}^{u, w-\varepsilon}(X)=$ $\operatorname{rk} \breve{H}_{0}^{u, w+\varepsilon}(X)=1$.

The second example, shown in Figure 5(b), is built in a similar way. The space $X$ is again obtained by adding an infinite number of branches to a vertical segment, but this time, the sequence of heights of their endpoints is $\left(2-\frac{1}{2^{n}}\right)_{n \in \mathbb{N}}$, converging to 2 , and $\varphi$ is again the height function. In this case, $w=2$ is a homological 0 critical value of $\varphi$. Indeed, for every sufficiently small $\varepsilon>0, \operatorname{rk} \breve{H}_{0}\left(X_{w-\varepsilon}\right)=2$ while $\operatorname{rk} \breve{H}_{0}\left(X_{w+\varepsilon}\right)=1$. On the other hand, for every $u<w$ and every small enough $\varepsilon>0$, it holds that $\operatorname{rk} \breve{H}_{0}^{u, w+\varepsilon}(X)=\operatorname{rk} \breve{H}_{0}^{u, w-\varepsilon}(X)=1$ or $\operatorname{rk} \breve{H}_{0}^{u, w+\varepsilon}(X)=\operatorname{rk} \breve{H}_{0}^{u, w-\varepsilon}(X)=0$. Therefore, in both cases, $\operatorname{rk} \breve{H}_{0}^{u, w-\varepsilon}(X)=\operatorname{rk} \breve{H}_{0}^{u, w+\varepsilon}(X)$ for every $u<w$ and every small enough $\varepsilon>0$, implying that $w$ is not the ordinate of any proper point of $D_{0}(X, \varphi)$ because of Lemma B.3(b). Moreover, we can immediately verify that, for every $v>w$ and every small enough $\varepsilon>0$, $\operatorname{rk} \breve{H}_{0}^{w+\varepsilon, v}(X)=\operatorname{rk} \check{H}_{0}^{w-\varepsilon, v}(X)=1$, i. e., by Lemma B.3(a), that $w$ is not the abscissa of a point of $D_{0}(X, \varphi)$.

\section{References}

[1] S. Biasotti, L. De Floriani, B. Falcidieno, P. Frosini, D. Giorgi, C. Landi, L. Papaleo, and M. Spagnuolo, Describing shapes by geometrical-topological properties of real functions, ACM Comput. Surv. $\mathbf{4 0}$ (2008), no. $4,1-87$.

[2] G. Carlsson, Topology and data, Bull. Amer. Math. Soc. 46 (2009), no. 2, 255-308.

[3] A. Cerri, B. Di Fabio, M. Ferri, P. Frosini, and C. Landi, Multidimensional persistent homology is stable, Technical report, Univ. of Bologna, 2009, http://amsacta.cib.unibo.it/2603/.

[4] J. Cho and J. Choi, Contour-based partial object recognition using symmetry in image databases, SAC '05: Proceedings of the 2005 ACM symposium on Applied computing, 2005, pp. 1190-1194.

[5] D. Cohen-Steiner, H. Edelsbrunner, and J. Harer, Stability of persistence diagrams, Discrete Comput. Geom. 37 (2007), no. 1, 103-120.

[6] M. d'Amico, P. Frosini, and C. Landi, Using matching distance in size theory: A survey, International Journal of Imaging Systems and Technology 16 (2006), no. 5, 154-161.

[7] __ Natural pseudo-distance and optimal matching between reduced size functions, Acta Applicandae Mathematicae 109 (2010), no. 2, 527-554.

[8] A. Delfinado and H. Edelsbrunner, An incremental algorithm for betti numbers of simplicial complexes, SCG '93: Proceedings of the ninth annual symposium on Computational geometry, 1993, pp. 232-239.

[9] H. Edelsbrunner and J. Harer, Persistent homology - a survey, Surveys on discrete and computational geometry, Contemp. Math., vol. 453, Amer. Math. Soc., Providence, RI, 2008, pp. 257-282.

[10] S. Eilenberg and N. Steenrod, Foundations of algebraic topology, Princeton University Press, Princeton, New Jersey, 1952.

[11] P. Frosini, Measuring shapes by size functions, Society of Photo-Optical Instrumentation Engineers (SPIE) Conference Series (D. P. Casasent, ed.), Presented at the Society of Photo-Optical Instrumentation Engineers (SPIE) Conference, vol. 1607, February 1992, pp. 122-133.

[12] P. Frosini and C. Landi, Size functions and formal series, Appl. Algebra Engrg. Comm. Comput. 12 (2001), no. $4,327-349$. 
[13] A. Ghosh and N. Petkov, Robustness of shape descriptors to incomplete contour representations, IEEE Transactions on Pattern Analysis and Machine Intelligence 27 (2005), 1793-1804.

[14] A. Hatcher, Algebraic topology, Cambridge University Press, 2002.

[15] J.-R. Hoynck, M. Ohm, Shape retrieval with robustness against partial occlusion, Proc. of IEEE Int. Conf. on Acoustics, Speech, and Signal Processing, 2003 (ICASSP '03), vol. 3, pp. 593-596.

[16] G. M. Kelly, The exactness of Čech homology over a vector space, Mathematical Proceedings of the Cambridge Philosophical Society 57 (1961), no. 02, 428-429.

[17] G. Kovács, R. Vogels, and G. A. Orban, Selectivity of macaque inferior temporal neurons for partially occluded shapes, The Journal of Neuroscience 15 (1995), no. 3, 1984-1997.

[18] C. Landi and P. Frosini, New pseudodistances for the size function space, Proceedings SPIE, Vision Geometry VI (Robert A. Melter, Angela Y. Wu, and Longin J. Latecki, eds.), vol. 3168, 1997, pp. 52-60.

[19] G. Mori, S. Belongie, and J. Malik, Shape contexts enable efficient retrieval of similar shapes, Computer Vision and Pattern Recognition, IEEE Computer Society Conference on 1 (2001), 723-730.

[20] http://www.imageprocessingplace.com/root_files_V3/image_databases.htm.

[21] K. B. Sun and B. J. Super, Classification of contour shapes using class segment sets, CVPR '05: Proceedings of the 2005 IEEE Computer Society Conference on Computer Vision and Pattern Recognition (CVPR'05), vol. 2, 2005, pp. 727-733.

[22] M. Tanase and R. C. Veltkamp, Part-based shape retrieval, MULTIMEDIA '05: Proceedings of the 13th annual ACM international conference on Multimedia, 2005, pp. 543-546.

[23] R. C. Veltkamp, Shape matching: Similarity measures and algorithms, Proc. of Shape Modeling International, 2001, pp. 188-197. 